\title{
THE OPTIMIZATION FOR THE INEQUALITIES OF POWER MEANS
}

\author{
JIAJIN WEN AND WAN-LAN WANG
}

Received 14 November 2005; Revised 5 May 2006; Accepted 14 July 2006

Let $M_{n}^{[t]}(a)$ be the $t$ th power mean of a sequence $a$ of positive real numbers, where $a=\left(a_{1}, a_{2}, \ldots, a_{n}\right), n \geq 2$, and $\alpha, \lambda \in \mathbb{R}_{++}^{m}, m \geq 2, \sum_{j=1}^{m} \lambda_{j}=1, \min \{\alpha\} \leq \theta \leq \max \{\alpha\}$. In this paper, we will state the important background and meaning of the inequality $\prod_{j=1}^{m}\left\{M_{n}^{\left[\alpha_{j}\right]}(a)\right\}^{\lambda_{j}} \leq(\geq) M_{n}^{[\theta]}(a)$; a necessary and sufficient condition and another interesting sufficient condition that the foregoing inequality holds are obtained; an open problem posed by Wang et al. in 2004 is solved and generalized; a rulable criterion of the semipositivity of homogeneous symmetrical polynomial is also obtained. Our methods used are the procedure of descending dimension and theory of majorization; and apply techniques of mathematical analysis and permanents in algebra.

Copyright (c 2006 J. Wen and W.-L. Wang. This is an open access article distributed under the Creative Commons Attribution License, which permits unrestricted use, distribution, and reproduction in any medium, provided the original work is properly cited.

\section{Symbols and introduction}

We will use some symbols in the well-known monographs $[1,5,13]$ :

$$
\begin{aligned}
& A_{n}=a=\left(a_{1}, \ldots, a_{n}\right), a^{\theta}=\left(a_{1}^{\theta}, \ldots, a_{n}^{\theta}\right), I_{n}=(1, \ldots, 1), O_{n}=(0, \ldots, 0), \\
& \alpha=\left(\alpha_{1}, \ldots, \alpha_{m}\right) ; \min \{\alpha\}=\min \left\{\alpha_{1}, \ldots, \alpha_{m}\right\} ; \max \{\alpha\}=\max \left\{\alpha_{1}, \ldots, \alpha_{m}\right\}, \\
& \lambda=\left(\lambda_{1}, \ldots, \lambda_{m}\right) ; \mathbb{R}^{n}=\left\{a: a_{i} \in \mathbb{R}, 1 \leq i \leq n\right\} ; \mathbb{R}_{+}^{n}=\left\{a: a_{i} \geq 0,1 \leq i \leq n\right\}, \\
& \mathbb{R}_{++}^{n}=\left\{a: a_{i}>0,1 \leq i \leq n\right\}, \mathbb{Z}_{+}^{n}=\left\{a \mid a_{i} \geq 0, a_{i} \text { is a integer, } i=1,2, \ldots, n\right\},(0,1]^{n}= \\
& \left\{a: 0<a_{i} \leq 1,1 \leq i \leq n\right\}, d \in \mathbb{R}, B_{d} \subset\left\{\alpha: \alpha \in \mathbb{R}^{m}, \alpha_{1}+\cdots+\alpha_{m}=d\right\}, B_{d} \text { is a finite set, }
\end{aligned}
$$
and it is not empty.

Recall that the definitions of the $t$ th power mean and Hardy mean of order $r$ for a sequence $a=\left(a_{1}, \ldots, a_{n}\right)(n \geq 2)$ are, respectively,

$$
\begin{aligned}
& M_{n}^{[t]}(a)=\left(\frac{1}{n} \cdot \sum_{i=1}^{n} a_{i}^{t}\right)^{1 / t}, \quad \text { if } 0<|t|<+\infty, \\
& M_{n}^{[t]}(a)=\sqrt[n]{a_{1} a_{2} \cdots a_{n}}, \quad \text { if } t=0,
\end{aligned}
$$


2 The optimization for the inequalities of power means

$$
\begin{aligned}
& H_{n}(a ; r)=\left[\frac{1}{n !} \cdot \sum_{i_{1}, \ldots, i_{n}} \prod_{j=1}^{n}\left(a_{i_{j}}\right)^{r_{j}}\right]^{1 /\left(r_{1}+\cdots+r_{n}\right)}, \quad \text { if } r_{1}+\cdots+r_{n}>0, \\
& H_{n}(a ; r)=\sqrt[n]{a_{1} a_{2} \cdots a_{n}}, \quad \text { if } r_{i}=0, i=1, \ldots, n,
\end{aligned}
$$

where $t \in \mathbb{R}, r \in \mathbb{R}_{+}^{n}, a \in \mathbb{R}_{++}^{n}$. And

$$
h_{n}(a ; r)=\frac{1}{n !} \cdot \sum_{i_{1}, \ldots, i_{n}} \prod_{j=1}^{n} a_{i_{j}}^{r_{j}}, \quad a \in \mathbb{R}_{++}^{n}, r \in \mathbb{R}^{n}
$$

is called Hardy function, where $i_{1}, \ldots, i_{n}$ is the total permutation of $1, \ldots, n$.

Definition 1.1. Let $\alpha \in \mathbb{R}^{n}$, let $\lambda_{\alpha}$ be a function of $\alpha, \lambda_{\alpha} \in \mathbb{R}, x \in \mathbb{R}_{++}^{n}$. Then the function $f(x)=\sum_{\alpha \in B_{d}} \lambda_{\alpha} h_{n}(x ; \alpha)$ is called the generalized homogeneous symmetrical polynomial of $n$ variables and degree $d$. When $B_{d} \subset Z_{+}^{n}, f(x)$ is called the homogeneous symmetrical polynomial of $n$ variables and degree $d$, simply, homogeneous symmetrical polynomial (see [24, page 431]).

Definition 1.2. Let $a_{i j}$ be the complex numbers, $i, j=1,2, \ldots, n$, and let the matrix $A=$ $\left(a_{i j}\right)_{n \times n}$ be an $n \times n$ matrix. Then the permanent (of order $n$ ) of $A$ is a function of matrix, written per $A$, it is defined by

$$
\operatorname{per} A=\sum_{\sigma} a_{1 \sigma_{1}} a_{2 \sigma_{2}} \cdots a_{n \sigma_{n}}
$$

where the summation extends over all one-to-one functions from $1, \ldots, n$ to $1, \ldots, n$. (See [12].) It is often convenient in the proof of Lemma 2.2 and Corollary 2.6 that we will also apply a symbol similar to determinant as follows:

$$
\operatorname{per} A=\left|\begin{array}{cccc}
a_{11} & a_{12} & \cdots & a_{1 n} \\
a_{21} & a_{22} & \cdots & a_{2 n} \\
\vdots & \vdots & \ddots & \vdots \\
a_{n 1} & a_{n 2} & \cdots & a_{n n}
\end{array}\right|_{n}^{+}
$$

It should be noted that the permanent remains some properties of the common determinant, but both of them are different. For example, for the common determinant, we have "the determinant changes sign if two adjacent rows are interchanged." But the affirmative proposition and its corollaries do not hold for permanents.

As pointed out in [1], the theory of inequalities plays an important role in all the fields of mathematics. And the power mean is the most important one in all the means. Many mathematicians wrote a great number of papers, and established the inequalities involving the power means and the related problems (see, e.g., $[1,4,5,8,9,13,14,17-$ $19,21])$. Recently, the authors studied the optimal real number $\lambda$ such that the following 
inequality:

$$
\left\{M_{n}^{[\alpha]}(a)\right\}^{1-\lambda}\left\{M_{n}^{[\beta]}(a)\right\}^{\lambda} \leq M_{n}^{[\theta]}(a)
$$

or its converse holds, where $a \in \mathbb{R}_{++}^{n}, 0<\alpha<\theta<\beta, \lambda \in \mathbb{R}$.

The optimal concepts are multifarious and versatile in mathematics (e.g., see $[8,19$, $20]$ ). Although it is so, the true worth for inequalities is as follows: if an inequality includes some parameters, we study that these parameters should satisfy some necessary and sufficient conditions such that this inequality holds, then we call that the inequality is optimized. In this paper, we want to discuss the following optimal problems that are more general inequalities than inequality (1.5).

Let $a \in \mathbb{R}_{++}^{n}, n \geq 2, \alpha, \lambda \in \mathbb{R}_{++}^{m}, m \geq 2, \sum_{j=1}^{m} \lambda_{j}=1, \min \{\alpha\} \leq \theta \leq \max \{\alpha\}$. A natural problem is the following: what are the necessary and sufficient conditions such that the inequalities

$$
\begin{aligned}
& \prod_{j=1}^{m}\left\{M_{n}^{\left[\alpha_{j}\right]}(a)\right\}^{\lambda_{j}} \leq M_{n}^{[\theta]}(a), \\
& \prod_{j=1}^{m}\left\{M_{n}^{\left[\alpha_{j}\right]}(a)\right\}^{\lambda_{j}} \geq M_{n}^{[\theta]}(a)
\end{aligned}
$$

hold, respectively?

Assume that the components of $a$ are complex numbers. Then inequality (1.6) (or (1.7)) can be expressed as

$$
\prod_{j=1}^{m}\left(\|a\|_{\alpha_{j}}\right)^{\lambda_{j}} \leq(\geq) C \cdot\|a\|_{\theta}
$$

where $\|a\|_{p}=\left(\sum_{i=1}^{n}\left|a_{i}\right|^{p}\right)^{1 / p}, p>1$ is the norm of $a$, and $C=n^{\sum_{j=1}^{m}\left(\lambda_{j} / \alpha_{j}\right)-1 / \theta}$.

Let $1 / p_{j}+1 / \alpha_{j}=1,1 / p+1 / \theta=1, p>1, p_{j}>1,1 \leq j \leq m$. If $f$ is a bounded linear functional on $\bigcap_{j=1}^{m} L^{p_{j}}[a, b] \cap \mathfrak{E}^{p}[a, b]$. From the well-known theorem (see, e.g., [26]), there exists a unique function $y(t) \in \bigcap_{j=1}^{m} L^{p_{j}}[a, b] \cap \mathcal{E}^{p}[a, b]$, such that $f(x)=$ $\int_{a}^{b} x(t) y(t) d t$. Thus we have

$$
\|f\|_{\alpha_{j}}=\|y\|_{\alpha_{j}}=\left(\int_{a}^{b}|y(t)|^{\alpha_{j}} d t\right)^{1 / \alpha_{j}}, \quad\|f\|_{\theta}=\|y\|_{\theta}=\left(\int_{a}^{b}|y(t)|^{\theta} d t\right)^{1 / \theta} .
$$

By the above facts, inequality (1.8) can also be expressed as

$$
\prod_{j=1}^{m}\left(\|f\|_{\alpha_{j}}\right)^{\lambda_{j}} \leq(\geq) C \cdot\|f\|_{\theta},
$$

where $C=(b-a)^{\sum_{j=1}^{m}\left(\lambda_{j} / \alpha_{j}\right)-1 / \theta}$.

Based on the above-mentioned definitions and the related depictions, an open problem posed in [19] and others, which will be solved in this paper, are significative. We 


\section{The optimization for the inequalities of power means}

obtain not only a necessary and sufficient condition, but also an interesting sufficient condition such that inequality (1.6) holds. Note that the inequalities (1.6), (1.8), and (1.10) play some roles in the geometry of convex body (see, e.g., [3, 7]). Our methods are, of late years, the approach of descending dimension and theory of majorization; and apply some techniques of mathematical analysis and permanents [12] in algebra. Note that the way of descending dimension used in this paper is different from $[15,23,25]$; and the majorization is an effective theory that "it can state the inwardness and the relation between the quantities" (see $[4,11,16]$ ). It is very interesting that the mathematical analysis and permanent can skillfully be combined.

\section{The background of inequality (1.6)}

The following theorem can display the background and meaning of inequality (1.6).

Theorem 2.1. Let $f(x)=\sum_{\alpha \in B_{d}} \lambda_{\alpha} h_{n}(x ; \alpha)\left(x \in \mathbb{R}_{++}^{n}\right)$ be a generalized homogeneous symmetrical polynomial of $n$ variables and degree $d$, where $d>0, B_{d} \subset \mathbb{R}_{+}^{n}, \lambda_{\alpha}>0$ (for all $\alpha \in B_{d}$ ), $\lambda=d^{-1} \cdot \alpha, \min \{\alpha\} \leq \theta \leq \max \{\alpha\}$ (for all $\alpha \in B_{d}$ ). If, for arbitrary $\alpha \in B_{d}$, $x \in \mathbb{R}_{++}^{n}$, the inequality

$$
\prod_{j=1}^{n}\left\{M_{n}^{\left[\alpha_{j}\right]}(x)\right\}^{\lambda_{j}} \leq M_{n}^{[\theta]}(x)
$$

holds, then, for arbitrary $x \in \mathbb{R}_{++}^{n}$,

$$
\left[\frac{f(x)}{f\left(I_{n}\right)}\right]^{1 / d} \leq M_{n}^{[\theta]}(x) .
$$

In particular, if $0<\theta \leq 1$, and the measurable set $G \subset \Omega_{n}:=\left\{x \mid \sum_{i=1}^{n} x_{i} \leq n, x \in \mathbb{R}_{+}^{n}\right\}$, then, for arbitrary real number $\delta>0$,

$$
\int_{G}\left[\frac{f(x)}{f\left(I_{n}\right)}\right]^{\delta} d x \leq \frac{n^{n}}{n !},
$$

where $d x=d x_{1} d x_{2} \cdots d x_{n}$.

LEMMA 2.2. If $0 \leq a_{i 1} \leq a_{i 2} \leq \cdots \leq a_{i n}, i=1,2, \ldots, n$, then

$$
\frac{1}{n !} \cdot\left|\begin{array}{cccc}
a_{11} & a_{12} & \cdots & a_{1 n} \\
a_{21} & a_{22} & \cdots & a_{2 n} \\
\vdots & \vdots & \ddots & \vdots \\
a_{n 1} & a_{n 2} & \cdots & a_{n n}
\end{array}\right|_{n}^{+} \leq \prod_{i=1}^{n} \frac{1}{n} \sum_{j=1}^{n} a_{i j} .
$$


Proof. We will prove the general case by the induction for $m$,

$$
\frac{1}{n !} \cdot\left|\begin{array}{cccc}
a_{11} & a_{12} & \cdots & a_{1 n} \\
\vdots & \vdots & \ddots & \vdots \\
a_{m 1} & a_{m 2} & \cdots & a_{m n} \\
1 & 1 & \cdots & 1 \\
\vdots & \vdots & \ddots & \vdots
\end{array}\right|_{n} \leq \prod_{i=1}^{m} \frac{1}{n} \sum_{j=1}^{n} a_{i j}
$$

All the elements of $n-m$ rows in the above permanent are 1 .

When $m=1$, then the sign of equality is valid in (2.5). Assume that $m=2$ below. We delete the element at $i$ th row and $j$ th column from the permanent per $A$, then we construct a permanent of order $n-1$, and it is called cofactor of $a_{i j}$ and is denoted by $M_{i j}$. Note the following identities and inequalities:

$$
\begin{gathered}
\frac{1}{(n-1) !} M_{1 j}=\frac{1}{n-1} \sum_{1 \leq k \leq n, k \neq j} a_{2 k}=\frac{1}{n-1}\left[\left(\sum_{k=1}^{n} a_{2 k}\right)-a_{2 j}\right], \\
\frac{1}{(n-1) !} M_{11} \geq \frac{1}{(n-1) !} M_{12} \geq \cdots \geq \frac{1}{(n-1) !} M_{1 n}, \quad a_{11} \leq a_{12} \leq \cdots \leq a_{1 n} .
\end{gathered}
$$

Therefore, the expansion of the permanent of the left-hand side of (2.5) in terms of elements of the first row is given by

$$
\text { the left-hand side of (2.5) } \begin{aligned}
& =\frac{1}{n} \cdot \sum_{j=1}^{n} a_{1 j} \cdot \frac{1}{(n-1) !} M_{1 j} \\
& \leq\left[\frac{1}{n} \cdot \sum_{j=1}^{n} a_{1 j}\right]\left[\frac{1}{n} \cdot \sum_{j=1}^{n} \frac{1}{(n-1) !} M_{1 j}\right]=\prod_{i=1}^{2} \frac{1}{n} \sum_{j=1}^{n} a_{i j},
\end{aligned}
$$

where we used Čebyšev's inequality.

Assume that the elements in the left-hand side of (2.5) are not all 1, and the count of these rows is equal to $m-1(m \geq 3)$, inequality (2.5) holds. We will prove that inequality (2.5) holds as follows.

First we prove that inequalities (2.6) hold still.

Note that the expansion of permanent $M_{1 j}$ in terms of elements of the first column is given by

$$
M_{11}=\left|\begin{array}{cccc}
a_{22} & a_{23} & \cdots & a_{2 n} \\
\vdots & \vdots & \ddots & \vdots \\
a_{m 2} & a_{m 3} & \cdots & a_{m n} \\
1 & 1 & \cdots & 1 \\
\vdots & \vdots & \ddots & \vdots
\end{array}\right|_{n-1}^{+}=\sum_{i=2}^{n} a_{i 2} M_{i 2}^{*}, \quad a_{i j}=1, \quad(m+1 \leq i \leq n, 1 \leq j \leq n) .
$$


6 The optimization for the inequalities of power means

Similarly,

$$
M_{12}=\left|\begin{array}{cccc}
a_{21} & a_{23} & \cdots & a_{2 n} \\
\vdots & \vdots & \ddots & \vdots \\
a_{m 1} & a_{m 3} & \cdots & a_{m n} \\
1 & 1 & \cdots & 1 \\
\vdots & \vdots & \ddots & \vdots
\end{array}\right|_{n-1}^{+}=\sum_{i=2}^{n} a_{i 1} M_{i 1}^{*}, \quad a_{i j}=1, \quad m+1 \leq i \leq n, 1 \leq j \leq n .
$$

Since $M_{i 1}^{*}=M_{i 2}^{*}>0,(i=2,3, \ldots, n)$, therefore, $M_{11}-M_{12}=\sum_{i=2}^{n}\left(a_{i 2}-a_{i 1}\right) M_{i 1}^{*} \geq 0$, namely,

$$
\frac{1}{(n-1) !} M_{11} \geq \frac{1}{(n-1) !} M_{12}
$$

Similarly,

$$
\frac{1}{(n-1) !} M_{12} \geq \frac{1}{(n-1) !} M_{13} \geq \cdots \geq \frac{1}{(n-1) !} M_{1 n}
$$

Thus, the first chain in (2.6) is proven; and the second chain of (2.6) is given.

By inequality (2.6) and Čebyšev's inequality, we obtain that

$$
\text { the left-hand side of } \begin{aligned}
(2.5) & =\frac{1}{n} \cdot \sum_{j=1}^{n} a_{1 j} \cdot \frac{1}{(n-1) !} M_{1 j} \\
& \leq\left[\frac{1}{n} \cdot \sum_{j=1}^{n} a_{1 j}\right]\left[\frac{1}{n} \cdot \sum_{j=1}^{n} \frac{1}{(n-1) !} M_{1 j}\right] .
\end{aligned}
$$

It is noteworthy that the sign of equality of (2.12) is valid when $a_{11}=a_{12}=\cdots=a_{1 n}=1$. If we change two rows (columns) in permanent, then permanent keeps invariable, then, from the assumption of the induction, we get

$$
\frac{1}{n} \cdot \sum_{j=1}^{n} \frac{1}{(n-1) !} M_{1 j}=\frac{1}{n !} \cdot\left|\begin{array}{cccc}
a_{21} & a_{23} & \cdots & a_{2 n} \\
\vdots & \vdots & \ddots & \vdots \\
a_{m 1} & a_{m 3} & \cdots & a_{m n} \\
1 & 1 & \cdots & 1 \\
\vdots & \vdots & \ddots & \vdots
\end{array}\right|_{n}^{+} \leq \prod_{i=2}^{m} \frac{1}{n} \cdot \sum_{j=1}^{n} a_{i j}
$$

From inequalities (2.12) and (2.13), we obtain inequality (2.5).

Letting $m=n$ in (2.5), we get inequality (2.4). So the proof is complete.

Lemma 2.3. If $x \in \mathbb{R}_{++}^{n}, \alpha \in B_{d} \subset \mathbb{R}_{+}^{n}, d>0, \lambda=d^{-1} \alpha, n \geq 2$, then

$$
H_{n}(x ; \alpha) \leq \prod_{j=1}^{n}\left\{M_{n}^{\left[\alpha_{j}\right]}(x)\right\}^{\lambda_{j}} .
$$


Proof. Just as well assume that $0<x_{1} \leq x_{2} \leq \cdots \leq x_{n}$, then

$$
0<x_{1}^{\alpha_{i}} \leq x_{2}^{\alpha_{i}} \leq \cdots \leq x_{n}^{\alpha_{i}}, \quad i=1,2, \ldots, n
$$

Thus, by the definition of permanent and by Lemma 2.2, we obtain that

$$
H_{n}(x ; \alpha) \leq\left[\prod_{i=1}^{n} \frac{1}{n} \cdot \sum_{j=1}^{n} x_{j}^{\alpha_{i}}\right]^{1 / d}=\prod_{j=1}^{n}\left\{M_{n}^{\left[\alpha_{j}\right]}(x)\right\}^{\lambda_{j}} .
$$

Proof of Theorem 2.1. By Lemma 2.3, we observe that

$$
\begin{aligned}
{\left[\frac{f(x)}{f\left(I_{n}\right)}\right]^{1 / d} } & =\left[\frac{\sum_{\alpha \in B_{d}} \lambda_{\alpha}\left(H_{n}(x ; \alpha)\right)^{d}}{\sum_{\alpha \in B_{d}} \lambda_{\alpha}}\right]^{1 / d} \leq\left[\frac{\sum_{\alpha \in B_{d}} \lambda_{\alpha} \prod_{j=1}^{n}\left(M_{n}^{\left[\alpha_{j}\right]}(x ; \alpha)\right)^{d \lambda_{j}}}{\sum_{\alpha \in B_{d}} \lambda_{\alpha}}\right]^{1 / d} \\
& \leq\left[\frac{\sum_{\alpha \in B_{d}} \lambda_{\alpha}\left(M_{n}^{[\theta]}(x ; \alpha)\right)^{d}}{\sum_{\alpha \in B_{d}} \lambda_{\alpha}}\right]^{1 / d}=M_{n}^{[\theta]}(x ; \alpha) .
\end{aligned}
$$

Clearly, $\left[f(x) / f\left(I_{n}\right)\right]^{\delta}$ is integrable on $G$. Therefore, by inequality (2.2), we obtain that

$$
\begin{aligned}
\int_{G}\left[\frac{f(x)}{f\left(I_{n}\right)}\right]^{\delta} d x & \leq \int_{\Omega_{n}}\left[\frac{f(x)}{f\left(I_{n}\right)}\right]^{\delta} d x \leq \int_{\Omega_{n}}\left\{M_{n}^{[\theta]}(x)\right\}^{\delta d} d x \\
& \leq \int_{\Omega_{n}}\left\{M_{n}^{[1]}(x)\right\}^{\delta d} d \mu \leq \int_{\Omega_{n}} 1^{\delta d} d x=\int_{\Omega_{n}} d x=\frac{n^{n}}{n !} .
\end{aligned}
$$

Remark 2.4. The literature [6] generalizes the well-known Hardy inequality

$$
\alpha \prec \beta \Longrightarrow h_{n}(x ; \alpha) \leq h_{n}(x ; \beta)
$$

to the convex functions, where $x \in \mathbb{R}_{++}^{n}, \alpha, \beta \in \mathbb{R}^{n}$; [24] generalizes the well-known Čebyšev inequality to the generalized homogeneous symmetrical polynomial; [22] studied a necessary and sufficient condition such that

$$
H_{n}(x ; \alpha) \leq H_{n}(x ; \beta)
$$

holds.

Remark 2.5. Lemma 2.2 is an important theorem. We can deduce an interesting conclusion from this fact as follows.

Corollary 2.6. Let $f(x)=\sum_{\alpha \in B_{d}} \lambda_{\alpha} h_{n}(x ; \alpha)\left(x \in \mathbb{R}_{++}^{n}\right)$ be a generalized homogeneous symmetrical polynomial of $n$ variables and degree $d$. If $d>0, B_{d} \subset \mathbb{R}_{+}^{n}, \lambda_{\alpha}>0$ (for all $\left.\alpha \in B_{d}\right),[1 / f(x)]^{\delta}$ is integrable on measurable set $G,(0,1]^{n} \subset G \subset \mathbb{R}_{++}^{n}$, then, for arbitrary 
8 The optimization for the inequalities of power means

real number $\delta>0$,

$$
\int_{G}\left[\frac{f\left(I_{n}\right)}{f(x)}\right]^{\delta} d x \geq\left\{\int_{0}^{1}\left[\frac{n}{t^{d}+n-1}\right]^{\delta} d t\right\}^{n},
$$

where $d x=d x_{1} d x_{2} \cdots d x_{n}$.

Proof. For all $\alpha \in B_{d}$, just as well assume that

$$
\alpha_{1} \geq \alpha_{2} \geq \cdots \geq \alpha_{n} \geq 0
$$

Then, when $x \in(0,1]^{n}$, we have

$$
0<x_{j}^{\alpha_{1}} \leq x_{j}^{\alpha_{2}} \leq \cdots \leq x_{j}^{\alpha_{n}}, \quad j=1,2, \ldots, n .
$$

From Lemma 2.2, we get

$$
h_{n}(x ; \alpha)=\frac{1}{n !} \cdot\left|\begin{array}{cccc}
x_{1}^{\alpha_{1}} & x_{1}^{\alpha_{2}} & \cdots & x_{1}^{\alpha_{n}} \\
x_{2}^{\alpha_{1}} & x_{2}^{\alpha_{2}} & \cdots & x_{2}^{\alpha_{n}} \\
\vdots & \vdots & \ddots & \vdots \\
x_{n}^{\alpha_{1}} & x_{n}^{\alpha_{2}} & \cdots & x_{n}^{\alpha_{n}}
\end{array}\right|_{n}^{+} \leq \prod_{i=1}^{n} \frac{1}{n} \cdot \sum_{j=1}^{n} x_{i}^{\alpha_{j}} .
$$

Since the exponential function $c^{t}(c>0)$ is a convex function on $\mathbb{R}$, therefore, by [16, page 59], we observe that $1 / n \cdot \sum_{j=1}^{n} x_{i}^{\alpha_{j}}$ is a Schur-convex function of $\alpha$ on $\mathbb{R}_{+}^{n}$. For all $\alpha \in \mathbb{R}_{+}^{n}$, $\alpha \prec\left(\sum_{j=1}^{n} \alpha_{j}, O_{n-1}\right)=\left(d, O_{n-1}\right)$ from which [16, page 54], we conclude that

$$
\begin{aligned}
g(\alpha) & :=\frac{1}{n} \cdot \sum_{j=1}^{n} x_{i}^{\alpha_{j}} \leq g\left(d, O_{n-1}\right)=\frac{x_{i}^{d}+n-1}{n}, \\
\frac{f\left(I_{n}\right)}{f(x)} & =\frac{\sum_{\alpha \in B_{d}} \lambda_{\alpha}}{\sum_{\alpha \in B_{d}} \lambda_{\alpha} h_{n}(x ; \alpha)} \\
& \geq \frac{\sum_{\alpha \in B_{d}} \lambda_{\alpha}}{\sum_{\alpha \in B_{d}} \lambda_{\alpha} \prod_{i=1}^{n}\left(x_{i}^{d}+n-1\right) / n}=\prod_{i=1}^{n} \frac{n}{x_{i}^{d}+n-1}, \\
\int_{G}\left(\frac{f\left(I_{n}\right)}{f(x)}\right)^{\delta} d x & \geq \int_{(0,1]^{n}}\left(\frac{f\left(I_{n}\right)}{f(x)}\right)^{\delta} d x \geq \int_{(0,1]^{n}}\left(\prod_{i=1}^{n} \frac{n}{x_{i}^{d}+n-1}\right)^{\delta} d x \\
& =\int_{(0,1]^{n}} \prod_{i=1}^{n}\left(\frac{n}{x_{i}^{d}+n-1}\right)^{\delta} d x=\int_{0}^{1} \int_{0}^{1} \cdots \int_{0}^{1} \prod_{i=1}^{n}\left(\frac{n}{x_{i}^{d}+n-1}\right)^{\delta} d x_{1} d x_{2} \cdots d x_{n} \\
& =\prod_{i=1}^{n} \int_{0}^{1}\left(\frac{n}{x_{i}^{d}+n-1}\right)^{\delta} d x_{i}=\left[\int_{0}^{1}\left(\frac{n}{t^{d}+n-1}\right)^{\delta} d t\right]^{n} .
\end{aligned}
$$


In Section 1 through Section 2, these pioneer studies that the authors attempted would demonstrate that these results of this paper occupy some important positions in the theory of inequalities, as well as they are often used in several function spaces.

\section{A necessary and sufficient condition that inequality (1.6) holds}

We have known from Section 2 that investigation that inequalities (1.6) and (1.7) hold has considerable meaning. In this section, we will discuss how to transform inequality (1.6) into an inequality involving fewer variables so that there is a possibility that inequality (1.6) can be proven by means of mathematical software.

Theorem 3.1. Let $a \in \mathbb{R}_{++}^{n}, \alpha, \lambda \in \mathbb{R}_{++}^{m}, n \geq m \geq 2, \sum_{j=1}^{m} \lambda_{j}=1, \min \{\alpha\} \leq \theta \leq \max \{\alpha\}$. Then, a necessary and sufficient condition such that inequality (1.6) holds is that inequality

$$
\prod_{j=1}^{m}\left\{M_{n}^{\left[\alpha_{j}\right]}\left(A_{m-1}, I_{k}, O_{n-m-k+1}\right)\right\}^{\lambda_{j}} \leq M_{n}^{[\theta]}\left(A_{m-1}, I_{k}, O_{n-m-k+1}\right)
$$

holds for all the $A_{m-1}=\left(a_{1}, a_{2}, \ldots, a_{m-1}\right) \in \mathbb{R}_{++}^{m-1}, k=0,1,2, \ldots, n-m+1$.

LEMMA 3.2. Let

$$
u(t)=\sum_{j=0}^{m} a_{j} t^{r_{j}}, \quad a_{j} \in \mathbb{R}-\{0\}, r_{j} \in \mathbb{R}, j=1,2, \ldots, m, m \geq 1, r_{0}=0, a_{0} \in \mathbb{R}, t \in \mathbb{R}_{++}^{1},
$$

be a common polynomial of one variable. Then $u(t)$ has at most $m$ zeroes on $\mathbb{R}_{++}^{1}$, that is, the count of elements of the set $U_{m}=\{t \mid u(t)=0, t>0\}$ is $\left|U_{m}\right|$, where $\left|U_{m}\right| \leq m$.

Proof. We will prove by means of the induction for $m$.

When $m=1$, the conclusion is clear. Assume that when $1 \leq k \leq m-1(m \geq 2)$, the inequality $\left|U_{k}\right| \leq k$ holds. We will prove that $\left|U_{m}\right| \leq m$ holds as follows. We can assume $r_{m}>r_{m-1}>\cdots>r_{1}, r_{j} \neq 0, j=1,2, \ldots, m$, then

$$
\begin{gathered}
u^{\prime}(t)=\sum_{j=0}^{m} r_{j} a_{j} t^{r_{j}-1}=t^{r_{1}-1} \cdot \sum_{j=1}^{m} r_{j} a_{j} t^{r_{j}-r_{1}}, \\
\left(r_{j} a_{j} \in \mathbb{R}-\{0\}, j=1,2, \ldots, m, m \geq 2, t \in \mathbb{R}_{++}^{1}\right) .
\end{gathered}
$$

Based on the assumption of induction, the common polynomial $\sum_{j=1}^{m} r_{j} a_{j} t^{r_{j}-r_{1}}$ has at most $m-1$ zeroes on $\mathbb{R}_{++}^{1}$. Since $t^{r_{1}-1}>0$, therefore $u^{\prime}(t)$ has at most $m-1$ zeroes on $\mathbb{R}_{++}^{1}, u(t)$ has at most $m-1$ extreme points on $\mathbb{R}_{++}^{1}$. Let all the extreme points of $u(t)$ on $\mathbb{R}_{++}^{1}$ be

$$
t_{1}, t_{2}, \ldots, t_{p}, \quad t_{1}<t_{2}<\cdots<t_{p}, 0 \leq p \leq m-1 .
$$

If $p=0$, then $u(t)$ is a monotonic function on $\mathbb{R}_{++}^{1}$. We may assume that $u(t)$ is a increasing function on $\mathbb{R}_{++}^{1}$. We will prove that $u(t)$ is a strictly increasing function on $\mathbb{R}_{++}^{1}$ as follows. 
Let $0<t_{1}^{\prime}<t_{2}^{\prime}$, then $u\left(t_{1}^{\prime}\right) \leq u\left(t_{2}^{\prime}\right)$. If $u\left(t_{1}^{\prime}\right)=u\left(t_{2}^{\prime}\right)$, then for all $t \in\left[t_{1}^{\prime}, t_{2}^{\prime}\right], u\left(t_{1}^{\prime}\right) \leq u(t) \leq$ $u\left(t_{2}^{\prime}\right)=u\left(t_{1}^{\prime}\right), u(t) \equiv u\left(t_{1}^{\prime}\right), u^{\prime}(t) \equiv 0$. Thus, for all $t \in\left[t_{1}^{\prime}, t_{2}^{\prime}\right], t$ is the zero of $u^{\prime}(t)$ on $\mathbb{R}_{++}^{1}$. This contradicts with $u^{\prime}(t)$ which has $m-1$ zeroes on $\mathbb{R}_{++}^{1}$. Therefore, $u\left(t_{1}^{\prime}\right)<u\left(t_{2}^{\prime}\right), u(t)$ is a strictly increasing function on $\mathbb{R}_{++}^{1}$. Based on these facts, the count of the zeroes of $u(t)$ on $\mathbb{R}_{++}^{1}$ is $\left|U_{m}\right| \leq 1 \leq m$.

If $p \geq 1$, we can assert by using the above method that $u(t)$ is a strictly monotonic function on each of the following $p+1$ intervals: $\left(0, t_{1}\right],\left[t_{1}, t_{2}\right], \ldots,\left[t_{p},+\infty\right)$. And the number of zeroes of $u(t)$ is at most 1 on each of these intervals, then the amount of zeroes of $u(t)$ on $\mathbb{R}_{++}^{1}$ is $\left|U_{m}\right| \leq p+1 \leq m$. This ends the proof of Lemma 3.2.

Lemma 3.3. Let $A_{n}=a \in \mathbb{R}_{+}^{n}, \alpha, \lambda \in \mathbb{R}_{++}^{m}, n \geq m \geq 2$. $F(a)$ denotes $\prod_{j=1}^{m}\left\{M_{n}^{\left[\alpha_{j}\right]}(a)\right\}^{\lambda_{j}}$. If $A_{q}$ is a critical point of $F\left(A_{q}, O_{n-q}\right.$ ) (for all $q: m \leq q \leq n$ ) on $D_{q}:=\left\{A_{q} \mid \sum_{r=1}^{q} a_{r}=\right.$ $\left.q, A_{q} \in \mathbb{R}_{+}^{q}\right\}$, then $a_{1}, a_{2}, \ldots, a_{q}$ satisfying $r \leq m$, where $m$ denotes largest number of the pair $\left(a_{i}, a_{j}\right)$ with $a_{i} \neq a_{j}, i<j$, for $i, j=1,2, \ldots, q$, that is, the amount of the elements in the set $\left\{a_{1}, a_{2}, \ldots, a_{q}\right\}$ is $\left|\left\{a_{1}, a_{2}, \ldots, a_{q}\right\}\right|$, and $\left|\left\{a_{1}, a_{2}, \ldots, a_{q}\right\}\right| \leq m$.

Proof. Make the Lagrange function $L=F\left(A_{q}, O_{n-q}\right)+\mu\left(\sum_{r=1}^{q} a_{r}-q\right)$. Then $A_{q}$ is a critical point of $F\left(A_{q}, O_{n-q}\right)$ on the domain $D_{q}$ if and only if $\partial L / \partial a_{k}=\partial F\left(A_{q}, O_{n-q}\right) /\left(\partial a_{k}\right)+$ $\mu=0, k=1,2, \ldots, q$, and $A_{q} \in D_{q}$. Since $\ln F\left(A_{q}, O_{n-q}\right)=\sum_{j=1}^{m} \ln \left\{M_{n}^{\left[\alpha_{j}\right]}\left(A_{q}, O_{n-q}\right)\right\}^{\lambda_{j}}=$ $\sum_{j=1}^{m}\left(\lambda_{j} / \alpha_{j}\right) \ln \left(\sum_{r=1}^{q} a_{r}^{\alpha_{j}} / n\right)$, therefore,

$$
\frac{\partial F\left(A_{q}, O_{n-q}\right)}{\partial a_{k}}\left[F\left(A_{q}, O_{n-q}\right)\right]^{-1}=\sum_{j=1}^{m} \frac{\lambda_{j}}{\alpha_{j}} \cdot \frac{\alpha_{j} \cdot a_{k}^{\alpha_{j}-1}}{\sum_{r=1}^{q} a_{r}^{\alpha_{j}}}=\sum_{j=1}^{m} \frac{\lambda_{j}}{\sum_{r=1}^{q} a_{r}^{\alpha_{j}}} \cdot a_{k}^{\alpha_{j}-1} .
$$

Let $A_{q}$ be a critical point of $F\left(A_{q}, O_{n-q}\right)$ on the domain $D_{q}$. We take the auxiliary function as follows:

$$
\begin{gathered}
u(t)=\sum_{j=0}^{m} b_{j} t^{r_{j}}: b_{j}=\frac{\lambda_{j} \cdot F\left(A_{q}, O_{n-q}\right)}{\sum_{r=1}^{q} a_{r}^{\alpha_{j}}} \neq 0, \\
r_{j}=\alpha_{j}-1 \in \mathbb{R}, \quad j=1,2, \ldots, m, \quad b_{0}=\mu .
\end{gathered}
$$

Then

$$
\begin{gathered}
\frac{\partial L}{\partial a_{k}}=0, \quad k=1,2, \ldots, q \Longleftrightarrow u\left(a_{k}\right)=0, \\
a_{k} \in U_{m}=\{t \mid u(t)=0, t>0\}, \quad k=1,2, \ldots, q \Longleftrightarrow\left\{a_{1}, a_{2}, \ldots, a_{q}\right\} \subset U_{m} .
\end{gathered}
$$

By Lemma 3.2, we obtain that $\left|\left\{a_{1}, a_{2}, \ldots, a_{q}\right\}\right| \leq\left|U_{m}\right| \leq m$. Lemma 3.3 is thus proved.

Proof of Theorem 3.1. Necessity. If inequality (1.6) holds, in (1.6), we put that $a=$ $\left(A_{m-1}, I_{k}, O_{n-m-k+1}\right)$, (for all $\left.k: 0 \leq k \leq n-m+1\right)$, then, (1.6) reduces to (3.1), thus (3.1) holds. 
Sufficiency. Assume that (3.1) holds. We will prove that inequality (1.6) holds.

Note that we will prove a more general conclusion, that is,

$$
\prod_{j=1}^{m}\left\{M_{n}^{\left[\alpha_{j}\right]}\left(A_{q}, O_{n-q}\right)\right\}^{\lambda_{j}} \leq M_{n}^{[\theta]}\left(A_{q}, O_{n-1}\right), \quad \forall q: m \leq q \leq n, \forall A_{q} \in \mathbb{R}_{+}^{q} .
$$

First we prove a special case $\theta=1$. Since both sides of (3.8) are a linear homogeneous function of $A_{q}$, therefore we may assume that $A_{q} \in D_{q}:=\left\{A_{q} \mid \sum_{r=1}^{q} a_{r}=q, A_{q} \in \mathbb{R}_{+}^{q}\right\}$. Thus, inequality (3.8) is equivalent to

$$
F\left(A_{q}, O_{n-q}\right) \leq \frac{q}{n}, \quad \forall q: m \leq q \leq n, \forall A_{q} \in D_{q},
$$

where the definitions of $F(a)$ and $D_{q}$ are in Lemma 3.3. We can prove that (3.9) holds for $q$ by the induction.

First we prove that (3.9) holds for the case $q=m$. If $a_{m}=0$, from (3.1), we get

$$
F\left(A_{q}, O_{n-q}\right)=F\left(A_{m-1}, I_{0}, O_{n-m+1}\right) \leq M_{n}^{[1]}\left(A_{m-1}, I_{0}, O_{n-m+1}\right)=\frac{q}{n},
$$

therefore (3.9) holds. Let $a_{m}>0$ below. Taking $k=1$ in (3.1), we have

$$
\prod_{j=1}^{m}\left\{M_{n}^{\left[\alpha_{j}\right]}\left(A_{m-1}, 1, O_{n-m}\right)\right\}^{\lambda_{j}} \leq M_{n}^{[1]}\left(A_{m-1}, 1, O_{n-m}\right) .
$$

Replacing $A_{m-1}$ by $A_{m-1} / a_{m}$ in (3.11), we obtain that

$$
\prod_{j=1}^{m}\left\{M_{n}^{\left[\alpha_{j}\right]}\left(\frac{A_{m-1}}{a_{m}}, 1, O_{n-m}\right)\right\}^{\lambda_{j}} \leq M_{n}^{[1]}\left(\frac{A_{m-1}}{a_{m}}, 1, O_{n-m}\right) .
$$

Multiplying both sides of (3.12) by $a_{m}$, then (3.12) reduces to (3.8), thus (3.9) holds.

Assume that we replace $q$ by $q-1(m+1 \leq q \leq n)$ in (3.9), we have (3.9). We will prove that (3.9) holds as follows. From the continuity and differentiability of $F\left(A_{q}, O_{n-q}\right)$ on $D_{q}$, we just have to prove that for the critical point $A_{q}$ of $F\left(A_{q}, O_{n-q}\right)$ on $D_{q}$, for the point $A_{q}$ on the boundary of $D_{q},(3.9)$ holds still.

Case 1. If $A_{q}$ is a critical point of $F\left(A_{q}, O_{n-q}\right)$ on $D_{q}$, from Lemma 3.3, we know that the amount of unequal terms of $a_{1}, a_{2}, \ldots, a_{q}$ is at most $m$.

By the symmetry, we may assume that $a_{m}=a_{m+1}=\cdots=a_{q}>0$. Thus, taking $k=$ $q-m+1$ in (3.1), we obtain that

$$
\begin{aligned}
F\left(A_{q}, O_{n-q}\right) & =F\left(A_{m-1}, a_{m} \cdot I_{q-m+1}, O_{n-q}\right)=a_{m} \cdot F\left(\frac{A_{m-1}}{a_{m}}, I_{q-m+1}, O_{n-q}\right) \\
& \leq a_{m} \cdot M_{n}^{[1]}\left(\frac{A_{m-1}}{a_{m}}, I_{q-m+1}, O_{n-q}\right)=M_{n}^{[1]}\left(A_{q}, O_{n-q}\right)=\frac{q}{n} .
\end{aligned}
$$

In other words, (3.9) holds. 
12 The optimization for the inequalities of power means

Case 2. Let $A_{q}$ be a point on the boundary of $D_{q}$. Then there exists a term in $a_{1}, a_{2}, \ldots, a_{q}$, this term must be zero. We may assume that $a_{q}=0$. From $A_{q} \in D_{q}, a_{1}+a_{2}+\cdots+a_{q-1}=$ $q$, $((q-1) / q) \cdot a_{1}+(q-1) / q \cdot a_{2}+\cdots+((q-1) / q) \cdot a_{q-1}=q-1$, therefore, if we take $X_{q-1}=((q-1) / q) \cdot A_{q-1}$, then $A_{q-1}=q /(q-1) \cdot X_{q-1}, X_{q-1} \in D_{q-1}$. Thus, by the assumption of induction, we obtain that

$$
\begin{aligned}
F\left(A_{q}, O_{n-q}\right) & =F\left(A_{q-1}, O_{n-q+1}\right)=F\left(\frac{q}{q-1} \cdot X_{q-1}, O_{n-q+1}\right) \\
& =\frac{q}{q-1} \cdot F\left(X_{q-1}, O_{n-q+1}\right) \\
& \leq \frac{q}{q-1} \cdot M_{n}^{[1]}\left(X_{q-1}, O_{n-q+1}\right) \\
& =\frac{q}{q-1} \cdot \frac{q-1}{n}=\frac{q}{n} .
\end{aligned}
$$

Based on the principle of induction, (3.9) has been proven.

Second, we will prove the general case $\theta \neq 1$. Letting

$$
\begin{aligned}
a^{\theta} & =\left(a_{1}^{\theta}, a_{2}^{\theta}, \ldots, a_{n}^{\theta}\right)=y=\left(y_{1}, y_{2}, \ldots, y_{n}\right) \\
& \in \mathbb{R}_{+}^{n} \Longleftrightarrow a=\left(y_{1}^{1 / \theta}, y_{2}^{1 / \theta}, \ldots, y_{n}^{1 / \theta}\right) \in \mathbb{R}_{+}^{n},
\end{aligned}
$$

then inequality (3.8) is equivalent to

$$
\prod_{j=1}^{m}\left\{M_{n}^{\left[\alpha_{j} / \theta\right]}\left(Y_{q}, O_{n-q}\right)\right\}^{\lambda_{j}} \leq M_{n}^{[1]}\left(Y_{q}, O_{n-q}\right), \quad \forall q: m \leq q \leq n, \forall A_{q} \in \mathbb{R}_{+}^{q} .
$$

Since $\alpha / \theta \in \mathbb{R}_{++}^{n}, \min \{\alpha / \theta\} \leq 1 \leq \max \{\alpha / \theta\}$, inequality (3.16) reduces to the case $\theta=1$, therefore inequality (3.16) holds.

Summarizing the above mentioned, inequality (3.8) has been proven. Taking $q=n$ in inequality (3.8), we obtain inequality (1.6). Theorem 3.1 is thus proved.

Corollary 3.4. Let $a \in \mathbb{R}_{++}^{n}, 0<\alpha<\theta<\beta, \lambda \in \mathbb{R}$. Then the maximal value of $\lambda$ such that inequality (1.5) holds is

$$
\lambda^{*}:=\inf _{t>0,0 \leq k \leq n-1}\left\{\frac{\ln M_{n}^{[\theta]}\left([a]_{t, n, k}\right)-\ln M_{n}^{[\alpha]}\left([a]_{t, n, k}\right)}{\ln M_{n}^{[\beta]}\left([a]_{t, n, k}\right)-\ln M_{n}^{[\alpha]}\left([a]_{t, n, k}\right)}\right\},
$$

where $[a]_{t, n, k}=\left(t, I_{k}, O_{n-k-1}\right)$. Inequality (1.5) holds if and only if $\lambda \leq \lambda^{*}$. 
Proof. By Theorem 3.1, inequality (1.5) holds if and only if

$$
\begin{aligned}
& \left\{M_{n}^{[\alpha]}\left([a]_{t, n, k}\right)\right\}^{1-\lambda}\left\{M_{n}^{[\beta]}\left([a]_{t, n, k}\right)\right\}^{\lambda} \\
& \quad \leq M_{n}^{[\theta]}\left([a]_{t, n, k}\right), \quad \forall t>0, \forall k: 0 \leq k \leq n-1, \\
& \quad \Longleftrightarrow \lambda \leq \frac{\ln M_{n}^{[\theta]}\left([a]_{t, n, k}\right)-\ln M_{n}^{[\alpha]}\left([a]_{t, n, k}\right)}{\ln M_{n}^{[\beta]}\left([a]_{t, n, k}\right)-\ln M_{n}^{[\alpha]}\left([a]_{t, n, k}\right)}, \quad \forall t>0, \forall k: 0 \leq k \leq n-1 \Longleftrightarrow \lambda \leq \lambda^{*} .
\end{aligned}
$$

Example 3.5. Let $\alpha=1 / 2, \theta=1, \beta=3 / 2, n=15$. By using (3.17) in Corollary 3.4, we have

$$
\begin{aligned}
\lambda^{*} & :=\inf _{t>0,0 \leq k \leq n-1}\left\{\frac{\ln M_{n}^{[\theta]}\left([a]_{t, n, k}\right)-\ln M_{n}^{[\alpha]}\left([a]_{t, n, k}\right)}{\ln M_{n}^{[\beta]}\left([a]_{t, n, k}\right)-\ln M_{n}^{[\alpha]}\left([a]_{t, n, k}\right)}\right\} \\
& =\inf _{t>0,0 \leq k \leq 14}\left\{\frac{\ln (t+k) / 15-2 \ln (\sqrt{t}+k) / 15}{(2 / 3) \ln \left(t^{3 / 2}+k\right) / 15-2 \ln (\sqrt{t}+k) / 15}\right\} .
\end{aligned}
$$

In fact, by means of Mathematica software, we can sketch the graphs of the functions of two variables $g(t, k):=(\ln ((t+k) / 15)-2 \ln ((\sqrt{t}+k) / 15)) /\left((2 / 3) \ln \left(\left(t^{3 / 2}+k\right) / 15\right)-\right.$ $2 \ln (\sqrt{t}+k) / 15)$ and $-g(t, k)$. Thus our problem can be explained from the graphs, our result is the following: if $a \in \mathbb{R}_{+}^{15}$, then $\lambda \leq 0.4160944179212302 \ldots$ if and only if

$$
\left\{M_{15}^{[1 / 2]}(a)\right\}^{1-\lambda}\left\{M_{15}^{[3 / 2]}(a)\right\}^{\lambda} \leq M_{15}^{[1]}(a)
$$

Remark 3.6. Corollary 3.4 is an open problem posed in [19].

In this section, we merely discuss the optimal problem of inequality (1.6) under the condition $n \geq m \geq 2$. When $m$ is sufficiently large, it is impossible that we apply Theorem 3.1 artificially. Owing to this reason, we will discuss the general case of inequality (1.6) in Section 4 . In other words, we will search for the necessary and sufficient condition such that $m \geq 2, n \geq 2$ hold. Our aim is to work artificially.

\section{The sufficient condition that inequality (1.6) holds}

Theorem 4.1. Let $\alpha \in \mathbb{R}_{++}^{m}, m \geq 2,0<\alpha_{1} \leq \cdots \leq \alpha_{p-1} \leq \theta \leq \alpha_{p} \leq \cdots \leq \alpha_{m}, 2 \leq p \leq m$, $\lambda \in \mathbb{R}_{++}^{m}, \sum_{j=1}^{m} \lambda_{j}=1$. If $\alpha_{m} \leq 2\left(\alpha_{p}+\theta\right)$, inf $t>0 \sum_{j=1}^{m}\left(\lambda_{j}\left(\theta-\alpha_{j}\right) /\left(2+(n-2) t^{\alpha_{j}}\right)\right) \geq 0$, then, for all $a \in \mathbb{R}_{++}^{n}, n \geq 2$, inequality (1.6) holds. 
14 The optimization for the inequalities of power means

Recall the definition (see, e.g., [5, pages 41-42] and $[9,19])$ of generalized logarithmic means $E(r, s ; x, y)$,

$$
E(r, s ; x, y)= \begin{cases}{\left[\frac{r}{s} \cdot \frac{y^{s}-x^{s}}{y^{r}-x^{r}}\right]^{1 /(s-r)},} & r s(r-s)(x-y) \neq 0, \\ {\left[\frac{1}{r} \cdot \frac{y^{r}-x^{r}}{\ln y-\ln x}\right]^{1 / r},} & s=0, r(x-y) \neq 0, \\ e^{-1 / r}\left[\frac{x^{x^{r}}}{y^{y^{r}}}\right]^{1 /\left(x^{r}-y^{r}\right)}, & r=s, r(x-y) \neq 0, \\ \sqrt{x y}, & r=s=0, x \neq y \\ x, & x=y .\end{cases}
$$

Lemma $4.2[9,19]$. Let $a_{1}$ and $a_{2}$ be two positive real numbers, and let $r, s, u, v$ be real numbers, where $r \neq s, u \neq v$. Then, for all the $a_{1}, a_{2}>0$, a necessary and sufficient condition such that the inequality

$$
E\left(r, s ; a_{1}, a_{2}\right) \leq E\left(u, v ; a_{1}, a_{2}\right)
$$

holds is that

$$
\begin{gathered}
r+s \leq u+v, \\
e(r, s) \leq e(u, v),
\end{gathered}
$$

where $0 \leq \min \{r, s, u, v\}$ or $\max \{r, s, u, v\} \leq 0$,

$$
e(x, y)= \begin{cases}\frac{x-y}{\ln x-\ln y}, & x y>0, x \neq y, \\ 0, & x y=0,\end{cases}
$$

when $\min \{r, s, u, v\}<0<\max \{r, s, u, v\}$,

$$
e(x, y)=\frac{|x|-|y|}{x-y}, \quad x, y \in \mathbb{R}, x \neq y .
$$

Lemma 4.3. Let $\alpha \in \mathbb{R}_{++}^{m}, m \geq 2,0<\alpha_{1} \leq \cdots \leq \alpha_{p-1} \leq 1 \leq \alpha_{p} \leq \cdots \leq \alpha_{m}, 2 \leq p \leq m$, $\lambda \in \mathbb{R}_{++}^{m}, \alpha_{m} \leq 2\left(\alpha_{p}+1\right)$. Define the function $\Phi: \mathbb{R}_{++}^{n} \rightarrow \mathbb{R}, \Phi(a):=-\ln \prod_{j=1}^{m}\left\{M_{n}^{\left[\alpha_{j}\right]}(a)\right\}^{\lambda_{j}}$, then a necessary and sufficient condition such that the function $\Phi$ is a Schur-convex function is that

$$
\inf _{t>0}\left\{\sum_{j=1}^{m} \frac{\lambda_{j}\left(1-\alpha_{j}\right)}{2+(n-2) t^{\alpha_{j}}}\right\} \geq 0 .
$$

Proof. From the literature $[11,16]$, we only have to prove that a necessary and sufficient condition such that the inequality

$$
\left(a_{1}-a_{2}\right)\left(\frac{\partial \Phi}{\partial a_{1}}-\frac{\partial \Phi}{\partial a_{2}}\right) \geq 0, \quad \forall a \in \mathbb{R}_{++}^{n},
$$

holds is that inequality (4.6) holds. 
Without loss of generality, we may assume that $a_{1}>a_{2}, 0<\alpha_{p-1}<1<\alpha_{p}$. Note that

$$
\begin{gathered}
\Phi(a)=-\sum_{j=1}^{m} \frac{\lambda_{j}}{\alpha_{j}} \ln \frac{\sum_{i=1}^{n} a_{i}^{\alpha_{j}}}{n}, \\
\frac{\partial \Phi}{\partial a_{1}}-\frac{\partial \Phi}{\partial a_{2}}=-\sum_{j=1}^{m} \frac{\lambda_{j}\left(a_{1}^{\alpha_{j}-1}-a_{2}^{\alpha_{j}-1}\right)}{\sum_{i=1}^{n} a_{i}^{\alpha_{j}}}, \quad a_{1}^{\alpha_{p}-1}-a_{2}^{\alpha_{p}-1}>0 .
\end{gathered}
$$

Thus, inequality (4.7) is equivalent to

$$
\sum_{j=1}^{m} \frac{\lambda_{j}}{\sum_{i=1}^{n} a_{i}^{\alpha_{j}}} \cdot \frac{a_{1}^{\alpha_{j}-1}-a_{2}^{\alpha_{j}-1}}{a_{1}^{\alpha_{p}-1}-a_{2}^{\alpha_{p}-1}} \leq 0
$$

Since

$$
\begin{aligned}
\frac{a_{1}^{\alpha_{j}-1}-a_{2}^{\alpha_{j}-1}}{a_{1}^{\alpha_{p}-1}-a_{2}^{\alpha_{p}-1}} & =\frac{a_{1}^{\alpha_{p} \cdot\left(\left(\alpha_{j}-1\right) / \alpha_{p}\right)}-a_{2}^{\alpha_{p} \cdot\left(\left(\alpha_{j}-1\right) / \alpha_{p}\right)}}{a_{1}^{\alpha_{p} \cdot\left(\left(\alpha_{p}-1\right) / \alpha_{p}\right)}-a_{2}^{\alpha_{p} \cdot\left(\left(\alpha_{p}-1\right) / \alpha_{p}\right)}} \\
& =\frac{\alpha_{j}-1}{\alpha_{p}-1} \cdot\left[E\left(\frac{\alpha_{j}-1}{\alpha_{p}}, \frac{\alpha_{p}-1}{\alpha_{p}} ; a_{1}^{\alpha_{p}}, a_{2}^{\alpha_{p}}\right)\right]^{\left(\alpha_{j}-\alpha_{p}\right) / \alpha_{p}}
\end{aligned}
$$

so, inequality (4.9) is equivalent to

$$
\sum_{j=1}^{m} \frac{\lambda_{j}\left(1-\alpha_{j}\right)}{\sum_{i=1}^{n} a_{i}^{\alpha_{j}}}\left[E\left(\frac{\alpha_{j}-1}{\alpha_{p}}, \frac{\alpha_{p}-1}{\alpha_{p}} ; a_{1}^{\alpha_{p}}, a_{2}^{\alpha_{p}}\right)\right]^{\left(\alpha_{j}-\alpha_{p}\right) / \alpha_{p}} \geq 0
$$

Sufficiency. Assume that (4.6) holds, we will prove that inequality (4.11) holds. In fact, we will follow every step in the following.

Step 1. We will prove that

$$
\frac{\lambda_{j}\left(1-\alpha_{j}\right)}{\sum_{i=1}^{n} a_{i}^{\alpha_{j}}} \geq \frac{\lambda_{j}\left(1-\alpha_{j}\right)}{2 u^{\alpha_{j}}+(n-2) v^{\alpha_{j}}}, \quad j=1,2, \ldots, m
$$

where $u=\left(\left(a_{1}^{\alpha_{p}}+a_{2}^{\alpha_{p}}\right) / 2\right)^{1 / \alpha_{p}}$, when $n>2$, we have $v=\left(\sum_{i=3}^{n} a_{i}^{\alpha_{p}} /(n-2)\right)^{1 / \alpha_{p}}$, when $n=2$, we may define an arbitrary value of $v$. Now we define that $v=u$. When $1 \leq j \leq p-1$, we have $\lambda_{j}\left(1-\alpha_{j}\right)>0,0<\alpha_{j}<\alpha_{p}$. Therefore, by the inequality with power means, we obtain that

$$
\sum_{i=1}^{n} a_{i}^{\alpha_{j}}=2 \cdot\left(\frac{a_{1}^{\alpha_{j}}+a_{2}^{\alpha_{j}}}{2}\right)^{\alpha_{j} / \alpha_{j}}+(n-2)\left(\frac{\sum_{i=3}^{n} a_{i}^{\alpha_{j}}}{n-2}\right)^{\alpha_{j} / \alpha_{j}} \leq 2 u^{\alpha_{j}}+(n-2) v^{\alpha_{j}}
$$

Thus, inequality (4.12) holds. When $j \geq p, \lambda_{j}\left(1-\alpha_{j}\right)<0, \alpha_{j} \geq \alpha_{p}>1$, the reverse inequality of (4.13) holds, therefore inequality (4.12) holds still. 
16 The optimization for the inequalities of power means

Step 2. We will prove that

$$
E\left(\frac{\alpha_{j}-1}{\alpha_{p}}, \frac{\alpha_{p}-1}{\alpha_{p}} ; a_{1}^{\alpha_{p}}, a_{2}^{\alpha_{p}}\right) \leq E\left(1,2 ; a_{1}^{\alpha_{p}}, a_{2}^{\alpha_{p}}\right)=\frac{a_{1}^{\alpha_{p}}+a_{2}^{\alpha_{p}}}{2}, \quad 1 \leq j \leq m, j \neq p .
$$

When $1 \leq j \leq p-1$,

$$
\begin{gathered}
\min \left\{\frac{\alpha_{j}-1}{\alpha_{p}}, \frac{\alpha_{p}-1}{\alpha_{p}}, 1,2\right\}=\frac{\alpha_{j}-1}{\alpha_{p}}<0<2=\max \left\{\frac{\alpha_{j}-1}{\alpha_{p}}, \frac{\alpha_{p}-1}{\alpha_{p}}, 1,2\right\}, \\
\frac{\alpha_{i}-1}{\alpha_{p}}+\frac{\alpha_{p}-1}{\alpha_{p}}<0+1<1+2, \\
\frac{\left|\left(\alpha_{j}-1\right) / \alpha_{p}\right|-\left|\left(\alpha_{p}-1\right) / \alpha_{p}\right|}{\left(\left(\alpha_{j}-1\right) / \alpha_{p}\right)-\left(\left(\alpha_{p}-1\right) / \alpha_{p}\right)}=\frac{2-\alpha_{p}-\alpha_{j}}{\alpha_{j}-\alpha_{p}}=\frac{2\left(1-\alpha_{j}\right)}{\alpha_{j}-\alpha_{p}}+1<1=\frac{|1|-|2|}{1-2},
\end{gathered}
$$

therefore, by Lemma 4.2, inequality (4.13) holds.

When $p+1 \leq j \leq m$,

$$
\begin{gathered}
\min \left\{\frac{\alpha_{j}-1}{\alpha_{p}}, \frac{\alpha_{p}-1}{\alpha_{p}}, 1,2\right\}=\frac{\alpha_{p}-1}{\alpha_{p}}>0, \\
\frac{\alpha_{j}-1}{\alpha_{p}}+\frac{\alpha_{p}-1}{\alpha_{p}}=\frac{\alpha_{j}+\alpha_{p}-2}{\alpha_{p}} \leq \frac{\alpha_{m}+\alpha_{p}-2}{\alpha_{p}} \leq \frac{2\left(\alpha_{p}+1\right)+\alpha_{p}-2}{\alpha_{p}}=1+2 .
\end{gathered}
$$

Based on the above discussion and Lemma 4.2, we only have to prove that

$$
\left(\frac{\alpha_{j}-1}{\alpha_{p}}-\frac{\alpha_{p}-1}{\alpha_{p}}\right) /\left(\ln \frac{\alpha_{j}-1}{\alpha_{p}}-\ln \frac{\alpha_{p}-1}{\alpha_{p}}\right) \leq \frac{1}{\ln 2}=\frac{1-2}{\ln 1-\ln 2} .
$$

Let $x=\ln \left(\left(\alpha_{j}-1\right) / \alpha_{p}\right), y=\ln \left(\left(\alpha_{p}-1\right) / \alpha_{p}\right)$, then $y \leq x \leq \ln \left(\left(\alpha_{m}-1\right) / \alpha_{p}\right) \leq \ln \left(\left(2 \alpha_{p}+\right.\right.$ $\left.1) / \alpha_{p}\right)=x_{0}$, from this fact, we get

$$
\begin{aligned}
& \left(\frac{\alpha_{j}-1}{\alpha_{p}}-\frac{\alpha_{p}-1}{\alpha_{p}}\right) /\left(\ln \frac{\alpha_{j}-1}{\alpha_{p}}-\ln \frac{\alpha_{p}-1}{\alpha_{p}}\right) \\
& =\frac{e^{x}-e^{y}}{x-y}=e^{y} \cdot \frac{e^{x-y}-1}{x-y}=e^{y} \cdot \sum_{k=0}^{\infty} \frac{(x-y)^{k}}{(k+1) !} \leq e^{y} \cdot \sum_{k=0}^{\infty} \frac{\left(x_{0}-y\right)^{k}}{(k+1) !} \\
& =\left(\frac{2 \alpha_{p}+1}{\alpha_{p}}-\frac{\alpha_{p}-1}{\alpha_{p}}\right) /\left(\ln \frac{2 \alpha_{p}+1}{\alpha_{p}}-\ln \frac{\alpha_{p}-1}{\alpha_{p}}\right) \\
& =\frac{1+2 t}{\ln (2+t)-\ln (1-t)}, \quad 0<t=\frac{1}{\alpha_{p}}<1 .
\end{aligned}
$$


Thus, we only have to prove that

$$
\begin{aligned}
\frac{1+2 t}{\ln (2+t)-\ln (1-t)} & \leq \frac{1}{\ln 2} \Longleftrightarrow \phi(t) \\
& :=\ln (2+t)-\ln (1-t)-(\ln 2)(1+2 t) \geq 0, \quad \forall t \in(0,1) .
\end{aligned}
$$

Since $\phi^{\prime}(t)=3 /\left(2-t-t^{2}\right)-2 \ln 2$ is increasing on $(0,1)$, we have

$$
\phi^{\prime}(t)>\phi^{\prime}(0)=\frac{3}{2}-2 \ln 2=0.11371 \cdots>0, \quad \phi(t)>\phi(0)=0 .
$$

It follows that inequality (4.17) and the assertion of Step 2 have been proven.

Step 3. We will prove that inequality (4.11) holds.

Since $\left(1-\alpha_{j}\right)\left(\alpha_{j}-\alpha_{p}\right)<0(j=1, \ldots, p-1, p+1, \ldots, m)$, therefore, by the inequalities (4.12) and (4.14), when $j \neq p$,

$$
\frac{\lambda_{j}\left(1-\alpha_{j}\right)}{\sum_{i=1}^{n} a_{i}^{\alpha_{j}}}\left[E\left(\frac{\alpha_{j}-1}{\alpha_{p}}, \frac{\alpha_{p}-1}{\alpha_{p}} ; a_{1}^{\alpha_{p}}, a_{2}^{\alpha_{p}}\right)\right]^{\left(\alpha_{j}-\alpha_{p}\right) / \alpha_{p}} \geq \frac{\lambda_{j}\left(1-\alpha_{j}\right) u^{\alpha_{j}-\alpha_{p}}}{2 u^{\alpha_{j}}+(n-2) v^{\alpha_{j}}},
$$

when $j=p$, inequality (4.21) reduces to the equality, so (4.21) holds still. Thus, letting $t=v / u>0$, we have

$$
\begin{aligned}
& \sum_{j=1}^{m} \frac{\lambda_{j}\left(1-\alpha_{j}\right)}{\sum_{i=1}^{n} a_{i}^{\alpha_{j}}}\left[E\left(\frac{\alpha_{j}-1}{\alpha_{p}}, \frac{\alpha_{p}-1}{\alpha_{p}} ; a_{1}^{\alpha_{p}}, a_{2}^{\alpha_{p}}\right)\right]^{\left(\alpha_{j}-\alpha_{p}\right) / \alpha_{p}} \\
& \geq \sum_{j=1}^{m} \frac{\lambda_{j}\left(1-\alpha_{j}\right) u^{\alpha_{j}-\alpha_{p}}}{2 u^{\alpha_{j}}+(n-2) v^{\alpha_{j}}}=u^{-\alpha_{p}} \sum_{j=1}^{m} \frac{\lambda_{j}\left(1-\alpha_{j}\right)}{2+(n-2) t^{\alpha_{j}}} \\
& \geq u^{-\alpha_{p}} \cdot \inf _{t>0} u^{-\alpha_{p}} \sum_{j=1}^{m} \frac{\lambda_{j}\left(1-\alpha_{j}\right)}{2+(n-2) t^{\alpha_{j}}} \geq 0 .
\end{aligned}
$$

It follows that inequality (4.11) holds.

Necessity. Assume that inequality (4.11) holds. We will prove that inequality (4.6) holds as follows.

Putting $a_{1}=a_{2}=1, a_{3}=\cdots=a_{n}=t>0$ in (4.11), then inequality (4.11) reduces to

$$
\sum_{j=1}^{m} \frac{\lambda_{j}\left(1-\alpha_{j}\right)}{2+(n-2) t^{\alpha_{j}}} \geq 0 \quad(\forall t>0) \Longrightarrow \inf _{t>0} \sum_{j=1}^{m} \frac{\lambda_{j}\left(1-\alpha_{j}\right)}{2+(n-2) t^{\alpha_{j}}} \geq 0 .
$$

This completes the proof.

Proof of Theorem 4.1. We first prove a special case $\theta=1$. By the hypothesis of Theorem 4.1 and Lemma 4.3 , the function

$$
\Phi: \mathbb{R}_{++}^{n} \longrightarrow \mathbb{R}, \quad \Phi(a):=-\ln \prod_{j=1}^{m}\left\{M_{n}^{\left[\alpha_{j}\right]}(a)\right\}^{\lambda_{j}}
$$

is a Schur-convex function. 
Let $A=\left(a_{1}+a_{2}+\cdots+a_{n}\right) / n$. Then $\bar{a}=(A, A, \ldots, A) \prec a$. From the definition of Schur-convex function, we observe that $\Phi(\bar{a}) \leq \Phi(a)$. By reason of $\lambda_{1}+\lambda_{2}+\cdots+\lambda_{n}=1$, it is easy to see that inequality (1.6) is equivalent to the inequality $\Phi(\bar{a}) \leq \Phi(a)$. Thus inequality (1.6) holds.

Second, we prove the general case $\theta \neq 1$ as follows.

By the hypothesis of Theorem 4.1, we obtain that

$$
\begin{gathered}
0<\frac{\alpha_{1}}{\theta} \leq \cdots \leq \frac{\alpha_{p-1}}{\theta} \leq 1 \leq \frac{\alpha_{p}}{\theta} \leq \cdots \leq \frac{\alpha_{m}}{\theta}, \quad \frac{\alpha_{m}}{\theta} \leq 2\left(\frac{\alpha_{p}}{\theta}+1\right), \\
\inf _{t>0} \sum_{j=1}^{m} \frac{\lambda_{j}\left(1-\alpha_{j} / \theta\right)}{2+(n-2) t^{\alpha_{j} / \theta}}=\frac{1}{\theta} \inf _{t^{1 / \theta}>0} \sum_{j=1}^{m} \frac{\lambda_{j}\left(\theta-\alpha_{j}\right)}{2+(n-2)\left(t^{1 / \theta}\right)^{\alpha_{j}}} \geq 0 .
\end{gathered}
$$

Combining the above with the conclusion of the special case $\theta=1$, we have

$$
\prod_{j=1}^{m}\left\{M_{n}^{\left[\alpha_{j} / \theta\right]}(a)\right\}^{\lambda_{j}} \leq M_{n}^{[1]}(a)
$$

Replacing $a$ by $a^{\theta}$ in (4.26), then inequality (4.26) reduces to inequality (1.6). This completes our proof.

Remark 4.4. Let $m=2$ in Theorem 4.1. Then we get [19, Theorem 1].

Corollary 4.5. If $a, b, x, y \in(0,+\infty)$, then

$$
\exp \frac{\int_{a}^{b}\left(\ln \left(x^{t}+y^{t}\right) / 2\right) \cdot(d t / t)}{b-a} \leq\left(\frac{x^{(a+b) / 2}+y^{(a+b) / 2}}{2}\right)^{2 /(a+b)}
$$

with equality holds if and only if $x=y$ or $a=b$.

Proof. In Theorem 4.1, letting $n=2, m=2 p-1, p \geq 2, \lambda_{j}=1 / m, \alpha_{j}=a+(j / m)(b-$ $a), b>a>0, j=1,2, \ldots, m,\left(a_{1}, a_{2}\right)=(x, y), \theta=(1 / m) \sum_{j=1}^{m} \alpha_{j}=a+(p / m)(b-a)=\alpha_{p}$, then we have $\alpha \in \mathbb{R}_{++}^{m}, m \geq 2,0<\alpha_{1} \leq \cdots \leq \alpha_{p-1} \leq \theta=\alpha_{p} \leq \cdots \leq \alpha_{m}, 2 \leq p \leq m, \lambda \in$ $\mathbb{R}_{++}^{m}, \sum_{j=1}^{m} \lambda_{j}=1$. Since

$$
\begin{gathered}
\alpha_{m}=b \leq b+a<2\left[a+\frac{p}{2 p-1}(b-a)\right]<2\left[a+\frac{p}{2 p-1}(b-a)+\theta\right]=2\left(\alpha_{p}+\theta\right), \\
\sum_{j=1}^{m} \frac{\lambda_{j}\left(\theta-\alpha_{j}\right)}{2+(n-2) t^{\alpha_{j}}}=\sum_{j=1}^{m} \frac{\lambda_{j}\left(\theta-\alpha_{j}\right)}{2}=0, \quad \inf _{t>0} \sum_{j=1}^{m} \frac{\lambda_{j}\left(\theta-\alpha_{j}\right)}{2+(n-2) t^{\alpha_{j}}} \geq 0,
\end{gathered}
$$


therefore, by Theorem 4.1 we have

$$
\begin{gathered}
\prod_{j=1}^{m}\left(\frac{x^{\alpha_{j}}+y^{\alpha_{j}}}{2}\right)^{1 / m \alpha_{j}} \leq\left(\frac{x^{\theta}+y^{\theta}}{2}\right)^{1 / \theta}, \\
\frac{1}{b-a} \sum_{j=1}^{m} \frac{1}{\alpha_{j}} \ln \left(\frac{x^{\alpha_{j}}+y^{\alpha_{j}}}{2}\right) \cdot \frac{b-a}{m} \leq \ln \left(\frac{x^{\theta}+y^{\theta}}{2}\right)^{1 / \theta}, \\
\frac{\int_{a}^{b}\left(\ln \left(x^{t}+y^{t}\right) / 2\right) \cdot d t / t}{b-a}=\lim _{m \rightarrow \infty} \frac{1}{b-a} \sum_{j=1}^{m} \frac{1}{\alpha_{j}} \ln \left(\frac{x^{\alpha_{j}}+y^{\alpha_{j}}}{2}\right) \cdot \frac{b-a}{m} \\
\leq \lim _{m \rightarrow \infty} \ln \left(\frac{x^{\theta}+y^{\theta}}{2}\right)^{1 / \theta}=\ln \left(\frac{x^{(a+b) / 2}+y^{(a+b) / 2}}{2}\right)^{2 /(a+b)} .
\end{gathered}
$$

In other words, inequality (4.27) has been proven. Corollary 4.5 is thus proved.

Example 4.6. Consider the condition such that the inequality

$$
\left\{\prod_{j=1}^{5} M_{10}^{[2 j]}(a)\right\}^{(1-\lambda) / 5}\left\{\prod_{j=6}^{10} M_{10}^{[2 j]}(a)\right\}^{\lambda / 5} \leq M_{10}^{[11]}(a), \quad \forall a \in \mathbb{R}_{++}^{10},
$$

holds.

Since $0<2<4<6<8<10<\theta=11<12<14<16<18<20<2(12+11)$, therefore, from Theorem 4.1, we know that, when

$$
\begin{gathered}
\inf _{t>0}\left\{\frac{1-\lambda}{5} \sum_{j=1}^{5} \frac{11-2 j}{2+8 t^{2 j}}+\frac{\lambda}{5} \sum_{j=6}^{10} \frac{11-2 j}{2+8 t^{2 j}}\right\} \geq 0 \Longleftrightarrow \lambda \leq \inf _{t>0}\{g(t)\}, \\
g(t)=\left(\sum_{j=1}^{5} \frac{11-2 j}{1+4 t^{2 j}}\right) /\left(\sum_{j=1}^{5} \frac{11-2 j}{1+4 t^{2 j}}+\sum_{j=6}^{10} \frac{2 j-11}{1+4 t^{2 j}}\right),
\end{gathered}
$$

inequality (4.30) holds. By means of Mathematica software, we can work out inf $g(t)=$ $0.297911 \ldots$. Namely, when $\lambda \leq 0.297911 \ldots$, inequality (4.30) holds.

\section{The necessary and sufficient condition that inequality (1.7) holds}

Theorem 5.1. Let $a \in \mathbb{R}_{++}^{n}, n \geq 2, \alpha, \lambda \in \mathbb{R}_{++}^{m}, m \geq 2, \sum_{j=1}^{m} \lambda_{j}=1, \min \{\alpha\} \leq \theta \leq \max \{\alpha\}$. Then, a necessary and sufficient condition such that inequality (1.7) holds is that

$$
\sum_{j=1}^{m} \frac{\lambda_{j}}{\alpha_{j}} \leq \frac{1}{\theta}
$$


Lemma 5.2. Let $a_{j k}>0, q_{j}>0, \sum_{j=1}^{m} q_{j} \leq 1,1 \leq j \leq m, 1 \leq k \leq n$. Then, an analogue of Hölder's inequality is

$$
\frac{1}{n} \cdot \sum_{k=1}^{n} \prod_{j=1}^{m} a_{j k}^{q_{j}} \leq \prod_{j=1}^{m}\left(\frac{1}{n} \cdot \sum_{k=1}^{n} a_{j k}\right)^{q_{j}}
$$

Proof of Theorem 5.1. Sufficiency. Assume that inequality (5.1) holds. We will prove that inequality (1.7) holds as follows: by (5.1), we have $\sum_{j=1}^{m}\left(\theta \lambda_{j} / \alpha_{j}\right) \leq 1, \theta \lambda_{j} / \alpha_{j}>0, j=$ $1,2, \ldots, m$. using Lemma 5.2 , we obtain that

$$
\begin{gathered}
{\left[\prod_{j=1}^{m}\left\{M_{n}^{\left[\alpha_{j}\right]}(a)\right\}^{\lambda_{j}}\right]^{\theta}=\prod_{j=1}^{m}\left(\frac{1}{n} \cdot \sum_{i=1}^{n} a_{i}^{\alpha_{j}}\right)^{\theta \lambda_{j} / \alpha_{j}} \geq \frac{1}{n} \cdot \sum_{i=1}^{n} \prod_{j=1}^{m}\left(a_{i}^{\alpha_{j}}\right)^{\theta \lambda_{j} / \alpha_{j}}} \\
=\frac{1}{n} \cdot \sum_{i=1}^{n} \prod_{j=1}^{m} a_{i}^{\theta \lambda_{j}}=\frac{1}{n} \cdot \sum_{i=1}^{n}\left(a_{i}\right)^{\theta \cdot \sum_{j=1}^{m} \lambda_{j}}=\frac{1}{n} \cdot \sum_{i=1}^{n}\left(a_{i}\right)^{\theta} .
\end{gathered}
$$

In other words, inequality (1.7) holds.

Necessity. Assume that inequality (1.7) holds. We will prove that inequality (5.1) holds as follows: letting $a_{1}=1, a_{2}=a_{3}=\cdots=a_{n} \rightarrow 0$ in inequality (1.7), (1.7) can be reduced to

$$
\prod_{j=1}^{m}\left\{\left(\frac{1}{n}\right)^{1 / \alpha_{j}}\right\}^{\lambda_{j}} \geq\left(\frac{1}{n}\right)^{1 / \theta} \Longleftrightarrow \prod_{j=1}^{m} n^{-\lambda_{j} / \alpha_{j}} \geq n^{-1 / \theta} \Longleftrightarrow n^{\sum_{j=1}^{m}\left(\lambda_{j} / \alpha_{j}\right)} \leq n^{1 / \theta} \Longleftrightarrow \sum_{j=1}^{m} \frac{\lambda_{j}}{\alpha_{j}} \leq \frac{1}{\theta} .
$$

Up to now, Theorem 5.1 is proven.

Remark 5.3. Applying the approach of [19], we can establish some results that are similar to $[19,(33)$ and $(37)]$. By using the definition of Riemann' integral, we can obtain an analogue of integral of (1.7) as follows.

Corollary 5.4. Let the measurable function on the measurable sets $E$ and $E_{0}$,

$$
f: E \longrightarrow \mathbb{R}_{++}^{1}, \quad g: E_{0} \longrightarrow \mathbb{R}_{++}^{1}, \quad p: E_{0} \longrightarrow \mathbb{R}_{++}^{1}, \quad E, E_{0} \subset \mathbb{R}^{n}
$$

satisfy that $\int_{E_{0}} p(t) d t=1, \inf _{t \in E_{0}} g(t) \leq \theta \leq \sup _{t \in E_{0}} g(t), \int_{E_{0}}(p(t) / g(t)) d t \leq 1 / \theta$. Then

$$
\exp \frac{\int_{E_{0}}(p(t) / g(t)) \ln \left\{\int_{E}[f(x)]^{g(t)} d x /|E|\right\} d t}{\left|E_{0}\right|} \geq\left\{\frac{\int_{E}[f(x)]^{\theta} d x}{|E|}\right\}^{1 / \theta}
$$

where $|E|$ and $\left|E_{0}\right|$ denote the measures of $E$ and $E_{0}$.

Inequality (5.6) has important background in the geometry of convex body (see, e.g., $[3,7])$. 


\section{The criterion of the semipositivity of homogeneous symmetric polynomial}

In this section, we will use the following symbols:

$$
\sigma_{k}=\sum_{1 \leq i_{1}<\cdots<i_{k} \leq n \leq n} \prod_{j=1}^{k} x_{i_{j}}, s_{k}=\sum_{i=1}^{n} x_{i}^{k}, A\left(x^{k}\right)=(1 / n) \cdot s_{k}, k=1,2, \ldots, d \wedge n, d \wedge
$$

$n=\min \{d, n\}, \overrightarrow{t(d \wedge n)}:=t(d \wedge n):=\left(t_{1}, \ldots, t_{d \wedge n}\right) \in Z_{+}^{d \wedge n}, \overrightarrow{d \wedge n} \cdot \overrightarrow{t(d \wedge n)}=\sum_{k=1}^{d \wedge n} k t_{k}$, $T_{d}=\left\{\overrightarrow{t(d \wedge n)} \mid \overrightarrow{d \wedge n} \cdot \overrightarrow{t(d \wedge n)}=d, \overrightarrow{t(d \wedge n)} \in Z_{+}^{d \wedge n}\right\}, \lambda=\left(\lambda_{1}, \ldots, \lambda_{i}, \ldots, \lambda_{d \wedge n}\right)=d^{-1} \cdot\left(t_{1}\right.$, $\left.2 t_{2}, \ldots, i t_{i}, \ldots, d \wedge n t_{d \wedge n}\right)$.

LEMMA 6.1. Let $f(x)=\sum_{\alpha \in B_{d}} \lambda_{\alpha} h_{n}(x ; \alpha)$ be a homogeneous symmetrical polynomial of $n$ ( $n$ $\geq 2$ ) variables of degree $d(d \geq 2)$, and let $f$ satisfy that $f\left(I_{n}\right)=0$. Then $f$ can be expressed as

$$
f(x)=\sum_{t(d \wedge n) \in T_{d, 1}} \lambda_{t(d \wedge n)}\left\{\prod_{i=1}^{d \wedge n}\left[M_{n}^{[i]}(x)\right]^{\lambda_{i}}\right\}^{d}-\sum_{t(d \wedge n) \in T_{d, 2}} \lambda_{t(d \wedge n)}\left\{\prod_{i=1}^{d \wedge n}\left[M_{n}^{[i]}(x)\right]^{\lambda_{i}}\right\}^{d},
$$

where $T_{d, 1}, T_{d, 2} \subset T_{d}, T_{d, 1} \cap T_{d, 2}=\Phi, T_{d, 1} \cup T_{d, 2}=T_{d}, \lambda_{t(d \wedge n)} \geq 0\left(\right.$ for all $\left.t(d \wedge n) \in T_{d}\right)$, and $\sum_{t(d \wedge n) \in T_{d, 1}} \lambda_{t(d \wedge n)}=\sum_{t(d \wedge n) \in T_{d, 2}} \lambda_{t(d \wedge n)}$.

Proof. By [2, Theorem 15, page 41], $f$ can be expressed as

$$
f(x)=\sum_{t(d \wedge n) \in T_{d}} \lambda_{t(d \wedge n)} \prod_{i=1}^{d \wedge n} \sigma_{i}^{t_{i}}
$$

Using Newton's formula (see[2, page 49] and [10, page 28]),

$$
\sigma_{0}=1, \quad \sum_{i=1}^{k}(-1)^{k-i} \sigma_{k-i} s_{i}+(-1)^{k} k \sigma_{k}=0, \quad 1 \leq k \leq n,
$$

or

$$
\sigma_{0}=1, \quad k ! \sigma_{k}=\left|\begin{array}{ccccc}
s_{1} & 1 & 0 & \cdots & 0 \\
s_{2} & s_{1} & 2 & \cdots & 0 \\
\vdots & \vdots & \vdots & \ddots & \vdots \\
s_{k-1} & s_{k-2} & s_{k-3} & \cdots & k-1 \\
s_{k} & s_{k-1} & s_{k-2} & \cdots & s_{1}
\end{array}\right|, \quad 1 \leq k \leq n,
$$

$\sigma_{i}$ can be expressed as

$$
\sigma_{i}=\sum_{t(i) \in T_{i}} \lambda_{t(i)} \prod_{j=1}^{i} s_{j}^{t_{j}} \quad(1 \leq i \leq n), \quad T_{i}=\left\{t(i) \mid \sum_{j=1}^{i} j t_{j}=i, t(i) \in Z_{+}^{i}\right\} .
$$


Equation (6.2) is substituted by (6.5); and using expansion formula for polynomial, $f$ can be expressed as

$$
\begin{aligned}
f(x) & =\sum_{t(d \wedge n) \in T_{d}} \lambda_{t(d \wedge n)}^{*} \prod_{i=1}^{d \wedge n} s_{i}^{t_{i}}=\sum_{t(d \wedge n) \in T_{d}} \lambda_{t(d \wedge n)} \prod_{i=1}^{d \wedge n} A^{t_{i}}\left(x^{i}\right) \\
& =\sum_{t(d \wedge n) \in T_{d}} \lambda_{t(d \wedge n)} \prod_{i=1}^{d \wedge n}\left\{M_{n}^{[i]}(x)\right\}^{i t_{i}}=\sum_{t(d \wedge n) \in T_{d}} \lambda_{t(d \wedge n)}\left\{\prod_{i=1}^{d \wedge n}\left[M_{n}^{[i]}(x)\right]^{\lambda_{i}}\right\}^{d} .
\end{aligned}
$$

Since $f\left(I_{n}\right)=0, \sum_{t(d \wedge n) \in T_{d}} \lambda_{t(d \wedge n)}=0$, by (6.6), there exist

$$
T_{d, 1}, T_{d, 2} \subset T_{d}, \quad T_{d, 1} \cap T_{d, 2}=\Phi, \quad T_{d, 1} \cup T_{d, 2}=T_{d}, \quad \lambda_{t(d \wedge n)} \geq 0 \quad\left(\forall t(d \wedge n) \in T_{d}\right),
$$

such that $f$ can be expressed as

$$
f(x)=\sum_{t(d \wedge n) \in T_{d, 1}} \lambda_{t(d \wedge n)}\left\{\prod_{i=1}^{d \wedge n}\left[M_{n}^{[i]}(x)\right]^{\lambda_{i}}\right\}^{d}-\sum_{t(d \wedge n) \in T_{d, 2}} \lambda_{t(d \wedge n)}\left\{\prod_{i=1}^{d \wedge n}\left[M_{n}^{[i]}(x)\right]^{\lambda_{i}}\right\}^{d},
$$

where $\sum_{t(d \wedge n) \in T_{d, 1}} \lambda_{t(d \wedge n)}=\sum_{t(d) \in T_{d, 2}} \lambda_{t(d \wedge n)}$. This completes the proof.

Theorem 6.2. Let $f(x)$ be a homogeneous symmetrical polynomial of $n(n \geq 2)$ variables of degree $d(d \geq n)$, and let $f\left(I_{n}\right)=0$, and let the expression of $f(x)$ be given by (6.1). The following can be written:

$$
\begin{aligned}
& \mu_{i}=\frac{\sum_{t(d \wedge n) \in T_{d, 1}} \lambda_{i} \lambda_{t(d \wedge n)}}{\sum_{t(d \wedge n) \in T_{d, 1}} \lambda_{t(d \wedge n)}}=\frac{\sum_{t(d \wedge n) \in T_{d, 1}} i t_{i} \lambda_{t(d \wedge n)}}{\sum_{t(d \wedge n) \in T_{d, 1}} d \cdot \lambda_{t(d \wedge n)}}, i=1,2, \ldots, d \wedge n, \\
& \theta_{1}=\left(\sum_{j=1}^{d \wedge n} \frac{\mu_{j}}{j}\right)^{-1}=\frac{d \cdot\left(\sum_{t(d \wedge n) \in T_{d, 1}} \lambda_{t(d \wedge n)}\right)}{\sum_{j=1}^{d \wedge n} \sum_{t(d \wedge n) \in T_{d, 1}} t_{j} \lambda_{t(d \wedge n)}}, \\
& \theta_{2}=\sup _{t>0, t(d \wedge n) \in T_{d, 2}}\left\{\left[\sum_{j=1}^{d \wedge n} \frac{j^{2} t_{j}}{2+(n-2) t^{j}}\right] /\left[\sum_{j=1}^{d \wedge n} \frac{j t_{j}}{2+(n-2) t^{j}}\right]\right\} .
\end{aligned}
$$

If, for arbitrary $t(d \wedge n) \in T_{d, 2}$, there exists $p: 2 \leq p \leq d \wedge n$ such that

$$
1 \leq \cdots \leq p-1 \leq \theta_{2} \leq p \leq \cdots \leq d \wedge n \leq 2\left(p+\theta_{2}\right)
$$

then, when $\theta_{1} \geq \theta_{2}$,

$$
f(x) \geq 0, \quad x \in \mathbb{R}_{++}^{n} .
$$


Proof. By the related theorem of continuous function, if $\lambda \in \mathbb{R}_{+}^{m}$ (or, $\lambda \in \mathbb{R}_{+}^{n}$ ), then the above theorem and lemma are valid.

By the arithmetic-geometric mean inequality and Theorem 5.1, we obtain that

$$
\begin{aligned}
& \sum_{t(d \wedge n) \in T_{d, 1}} \lambda_{t(d \wedge n)}\left\{\prod_{i=1}^{d \wedge n}\left[M_{n}^{[i]}(x)\right]^{\lambda_{i}}\right\}^{d} \\
& \geq\left(\sum_{t(d \wedge n) \in T_{d, 1}} \lambda_{t(d \wedge n)}\right)\left\{\prod_{t(d \wedge n) \in T_{d, 1}}\left[\prod_{i=1}^{d \wedge n}\left(M_{n}^{[i]}(x)\right)^{\lambda_{i}}\right]^{d \cdot \lambda_{t(d \wedge n)}}\right\}^{1 / \sum_{t(d \wedge n) \in T_{d, 1}} \lambda_{t(d \wedge n)}} \\
& =\left(\sum_{t(d \wedge n) \in T_{d, 1}} \lambda_{t(d \wedge n)}\right)\left\{\prod_{i=1}^{d \wedge n} \prod_{t(d \wedge n) \in T_{d, 1}}\left[\left(M_{n}^{[i]}(x)\right)^{\lambda_{i}}\right]^{d \cdot \lambda_{t(d \wedge n)}}\right\}^{1 / \sum_{t(d \wedge n) \in T_{d, 1}} \lambda_{t(d \wedge n)}} \\
& =\left(\sum_{t(d \wedge n) \in T_{d, 1}} \lambda_{t(d \wedge n)}\right)\left\{\prod_{i=1}^{d \wedge n}\left[M_{n}^{[i]}(x)\right]^{\sum_{t(d \wedge n) \in T_{d, 1}} \lambda_{i} \cdot d \cdot \lambda_{t(d \wedge n)}}\right\}^{1 / \sum_{t(d \wedge n) \in T_{d, 1}} \lambda_{t(d \wedge n)}} \\
& =\left(\sum_{t(d \wedge n) \in T_{d, 1}} \lambda_{t(d \wedge n)}\right)\left\{\prod_{i=1}^{d \wedge n}\left[M_{n}^{[i]}(x)\right]^{\mu_{i}}\right\}^{d} \geq\left(\sum_{t(d \wedge n) \in T_{d, 1}} \lambda_{t(d \wedge n)}\right)\left\{M_{n}^{\left[\theta_{1}\right]}(x)\right\}^{d} .
\end{aligned}
$$

By the definition of $\theta_{2}$ and $\lambda=d^{-1} \cdot\left(t_{1}, 2 t_{2}, \ldots, j t_{j}, \ldots, d \wedge n t_{d \wedge n}\right), \overrightarrow{d \wedge n} \cdot \overrightarrow{t(d \wedge n)}=d$, for for all $t(d \wedge n) \in T_{d, 2}$, we have $\inf _{t>0}\left\{\sum_{j=1}^{d \wedge n}\left(\lambda_{j}\left(\theta_{2}-j\right) /\left(2+(n-2) t^{j}\right)\right)\right\} \geq 0$, and by the hypothesis of Theorems 4.1 and 6.2, for all $t(d \wedge n) \in T_{d, 2}$, we have

$$
\begin{aligned}
& \sum_{t(d \wedge n) \in T_{d, 2}} \lambda_{t(d \wedge n)}\left\{\prod_{i=1}^{d \wedge n}\left[M_{n}^{[i]}(x)\right]^{\lambda_{i}}\right\}^{d} \\
& \quad \leq \sum_{t(d \wedge n) \in T_{d, 2}} \lambda_{t(d \wedge n)}\left\{M_{n}^{\left[\theta_{2}\right]}(x)\right\}^{d}=\left(\sum_{t(d \wedge n) \in T_{d, 2}} \lambda_{t(d \wedge n)}\right)\left\{M_{n}^{\left[\theta_{2}\right]}(x)\right\}^{d} .
\end{aligned}
$$

By (6.1), (6.12), (6.13), $\theta_{1} \geq \theta_{2}$, and $\sum_{t(d \wedge n) \in T_{d, 1}} \lambda_{t(d \wedge n)}=\sum_{t(d \wedge n) \in T_{d, 2}} \lambda_{t(d \wedge n)}$, inequality (6.11) holds. This completes the proof.

Example 6.3. Consider the condition such that the following inequality holds:

$$
\begin{aligned}
(1-s) & \left(\frac{1}{10} \sum_{i=1}^{10} x_{i}^{6}\right)\left(\frac{1}{10} \sum_{i=1}^{10} x_{i}^{10}\right)^{2}+s\left(\frac{1}{10} \sum_{i=1}^{10} x_{i}^{2}\right)^{3}\left(\frac{1}{10} \sum_{i=1}^{10} x_{i}^{4}\right)^{5} \\
- & \frac{1}{2}\left[\left(\frac{1}{10} \sum_{i=1}^{10} x_{i}\right)^{10}\left(\frac{1}{10} \sum_{i=1}^{10} x_{i}^{4}\right)^{4}+\left(\frac{1}{10} \sum_{i=1}^{10} x_{i}^{2}\right)^{9}\left(\frac{1}{10} \sum_{i=1}^{10} x_{i}^{8}\right)\right] \geq 0,
\end{aligned}
$$


24 The optimization for the inequalities of power means

where $d=26, n=10, d \wedge n=10$,

$$
\begin{gathered}
T_{d, 1}=\{(0,0,0,0,0,1,0,0,0,2),(0,3,0,5,0,0,0,0,0,0)\}, \\
T_{d, 2}=\{(10,0,0,4,0,0,0,0,0,0),(0,9,0,0,0,0,0,1,0,0)\}, \\
\theta_{1}=\left(\sum_{j=1}^{d \wedge n} \frac{\mu_{j}}{j}\right)^{-1}=\frac{d \cdot\left(\sum_{t(d \wedge n) \in T_{d, 1}} \lambda_{t(d \wedge n)}\right)}{\sum_{j=1}^{d \wedge n} \sum_{t(d \wedge n) \in T_{d, 1}} t_{j} \lambda_{t(d \wedge n)}}=\frac{26 \times 1}{(1-s)+2(1-s)+3 s+5 s}=\frac{26}{3+5 s}, \\
\theta_{2}=\sup _{t>0, t(d \wedge n) \in T_{d, 2}}\left\{\left[\sum_{j=1}^{d \wedge n} \frac{j^{2} t_{j}}{2+(n-2) t^{j}}\right] /\left[\sum_{j=1}^{d \wedge n} \frac{j t_{j}}{2+(n-2) t^{j}}\right]\right\} \\
=\sup _{t>0, t(10) \in T_{d, 2}}\left\{\left[\sum_{j=1}^{10} \frac{j^{2} t_{j}}{2+8 t^{j}}\right] /\left[\sum_{j=1}^{10} \frac{j t_{j}}{2+8 t^{j}}\right]\right\} \\
=\sup _{t>0, t(10) \in T_{d, 2}}\left\{\left[\frac{5}{1+4 t}+\frac{32}{1+4 t^{4}}\right] /\left[\frac{5}{1+4 t}+\frac{8}{1+4 t^{4}}\right],\left[\frac{18}{1+4 t}+\frac{32}{1+4 t^{4}}\right] /\left[\frac{9}{1+4 t}+\frac{4}{1+4 t^{4}}\right]\right\} .
\end{gathered}
$$

Using Mathematica software, we obtain that $\theta_{2}=510279565184453 \ldots$...

It follows that

$$
\theta_{1} \geq \theta_{2} \Longleftrightarrow \frac{26}{3+5 s} \geq \theta_{2} \Longleftrightarrow s \leq \frac{1}{5}\left(\frac{26}{\theta_{2}}-3\right)=0.41904923394695076 \ldots
$$

Namely, for $0<s \leq 0.41904923394695076 \ldots$, inequality (6.14) holds.

Remark 6.4. It must be pointed out that Theorem 6.2 can be operated artificially. Theorem 6.2 is different from the result in [15], because that of [15] only has meaning for $n \geq[d / 2]$ (i.e., the greatest integer function of $d / 2$ ) and can be operated artificially. The problem in Example 6.3 is too difficult, and furthermore, it cannot be solved by all the softwares in the existing circumstances.

\section{Acknowledgments}

The authors are very grateful to Professors Siegfried Carl, Enhao Yang, Tao Lu, and some referees for their encouragement and guidance, and would like also to acknowledge Support no. 10171073 from the NSF of China.

\section{References}

[1] P. S. Bullen, D. S. Mitrinović, and P. M. Vasić, Means and Their Inequalities, Mathematics and Its Applications (East European Series), vol. 31, D. Reidel, Dordrecht, 1988.

[2] Department of Mathematics Mechanics of Beijing University, Higher Algebra, People's Education Press, Beijing, 1978.

[3] R. J. Gardner, The Brunn-Minkowski inequality, Bulletin of the American Mathematical Society. New Series 39 (2002), no. 3, 355-405.

[4] K. Guan, Schur-convexity of the complete elementary symmetric function, Journal of Inequalities and Applications 2006 (2006), Article ID 67624, 9 pages. 
[5] J. C. Kuang, Applied Inequalities, Hunan Education Press, Changsha, 2004.

[6] L. Lai and J. J. Wen, Generalization for Hardy's inequality of convex function, Journal of Southwest University for Nationalities (Natural Science Edition) 29 (2003), no. 3, 269-274 (Chinese).

[7] G. Leng, C. Zhao, B. He, and X. Li, Inequalities for polars of mixed projection bodies, Science in China. Series A. Mathematics 47 (2004), no. 2, 175-186.

[8] T. P. Lin, The power mean and the logarithmic mean, The American Mathematical Monthly 81 (1974), no. 8, 879-883.

[9] Z. Liu, Comparison of some means, Journal of Mathematical Research and Exposition 22 (2002), no. $4,583-588$.

[10] I. G. Macdonald, Symmetric Functions and Hall Polynomials, 2nd ed., Oxford Mathematical Monographs, The Clarendon Press, Oxford University Press, New York, 1995.

[11] A. W. Marshall and I. Olkin, Inequalities: Theory of Majorization and Its Applications, Mathematics in Science and Engineering, vol. 143, Academic Press, New York, 1979.

[12] H. Minc, Permanents, Addison-Wesley, Massachusetts, 1988.

[13] D. S. Mitrinović, J. E. Pečarić, and A. M. Fink, Classical and New Inequalities in Analysis, Mathematics and Its Applications (East European Series), vol. 61, Kluwer Academic, Dordrecht, 1993.

[14] J. E. Pečarić and D. Svrtan, New refinements of the Jensen inequalities based on samples with repetitions, Journal of Mathematical Analysis and Applications 222 (1998), no. 2, 365-373.

[15] V. Timofte, On the positivity of symmetric polynomial functions. I. General results, Journal of Mathematical Analysis and Applications 284 (2003), no. 1, 174-190.

[16] B. Y. Wang, An Introduction to the Theory of Majorizations, Beijing Normal University Press, Beijing, 1990.

[17] Z. L. Wang and X. H. Wang, Quadrature formula and analytic inequalities-on the separation of power means by logarithmic mean, Journal of Hangzhou University 9 (1982), no. 2, 156-159.

[18] W.-L. Wang and P. F. Wang, A class of inequalities for the symmetric functions, Acta Mathematica Sinica 27 (1984), no. 4, 485-497 (Chinese).

[19] W.-L. Wang, J. J. Wen, and H. N. Shi, Optimal inequalities involving power means, Acta Mathematica Sinica 47 (2004), no. 6, 1053-1062 (Chinese).

[20] Z. Wei, L. Qi, and J. R. Birge, A new method for nonsmooth convex optimization, Journal of Inequalities and Applications 2 (1998), no. 2, 157-179.

[21] J. J. Wen, The optimal generalization of A-G-H inequalities and its applications, Journal of Shaanxi Normal University (2004), 12-16 (Chinese).

[22] Hardy means and their inequalities, to appear in Journal of Mathematics.

[23] J. J. Wen, W.-L. Wang, and Y. J. Lu, The method of descending dimension for establishing inequalities, Journal of Southwest University for Nationalities 29 (2003), no. 5, 527-532 (Chinese).

[24] J. J. Wen, C. J. Xiao, and R. X. Zhang, Chebyshev's inequality for a class of homogeneous and symmetric polynomials, Journal of Mathematics 23 (2003), no. 4, 431-436 (Chinese).

[25] J. J. Wen, R. X. Zhang, and Y. Zhang, Inequalities involving the means of variance and their applications, Journal of Sichuan University. Natural Science Edition 40 (2003), no. 6, 1011-1018 (Chinese).

[26] W. X. Zheng and S. W. Wang, An Introduction to Real and Functional Analysis (no.2), People's Education Press, Shanghai, 1980.

Jiajin Wen: Department of Mathematics and Computer Science, Chengdu University,

Chengdu 610106, Sichuan, China

E-mail address: wenjiajin623@163.com

Wan-Lan Wang: Department of Mathematics and Computer Science, Chengdu University, Chengdu 610106, Sichuan, China

E-mail address: wanlanwang@163.com 